\title{
An Axiomatic Analysis of Joint Liability Problems with Rooted -Tree Structure
}

Takayuki Oishia

Gerard van der Laan ${ }^{b}$

René van den Brink ${ }^{b}$

a Department of Economics, Aomori Public University, Japan;

b Faculty of Economics and Business Administration, VU University Amsterdam, and Tinbergen Institute, the Netherlands. 
Tinbergen Institute is the graduate school and research institute in economics of Erasmus University Rotterdam, the University of Amsterdam and VU University Amsterdam.

More TI discussion papers can be downloaded at http://www.tinbergen.nl

Tinbergen Institute has two locations:

Tinbergen Institute Amsterdam

Gustav Mahlerplein 117

1082 MS Amsterdam

The Netherlands

Tel.: +31(0)20525 1600

Tinbergen Institute Rotterdam

Burg. Oudlaan 50

3062 PA Rotterdam

The Netherlands

Tel.: +31(0)10 4088900

Fax: +31(0)10 4089031 


\title{
An axiomatic analysis of joint liability problems with rooted-tree structure ${ }^{1}$
}

\author{
Takayuki Oishi ${ }^{2} \quad$ Gerard van der Laan ${ }^{3} \quad$ René van den Brink ${ }^{4}$
}

May 25, 2016

\footnotetext{
${ }^{1}$ The authors would like to thank Shin Sakaue for his helpful comments.

${ }^{2}$ Department of Economics, Aomori Public University, e-mail: takayuki1q80@gmail.com

${ }^{3}$ Department of Econometrics and Operations Research, and Tinbergen Institute, VU University Amsterdam, e-mail: g.vander.laan@vu.nl

${ }^{4}$ Department of Econometrics and Operations Research, and Tinbergen Institute, VU University Amsterdam, e-mail: j.r.vanden.brink@vu.nl
} 


\begin{abstract}
For joint liability problems concerning tort law, a legal compensation scheme may be based on lower and upper bounds of compensation for injury and on case-system consistency. Introducing several properties inspired from this observation, we analyze compensation schemes axiomatically under the situation where causation of the cumulative injury appears in multiple sequences of wrongful acts. The situation underlying the model is described by a rooted-tree graph. We show that there is a unique compensation scheme that satisfies three axioms, one about lower bounds of individual compensations, one about upper bounds of individual compensations, and one about case-system consistency. This unique compensation scheme is the nucleolus of an associated liability game.
\end{abstract}

Keywords: Liability problems, Tort law, Rooted-tree graph, Axiomatization, Nucleolus, JEL Classification Number: D63, K13, K49 


\section{Introduction}

In this paper we consider situations in which an injured party suffers damages caused by wrongful acts performed subsequently by a sequence of injuring parties. The wrongful acts are causally related in the sense that any wrongful act in the sequence would not have occurred if any of the preceding wrongful acts would not have occurred. So, the second (wrongful) act can only occur after the first (wrongful) act has occurred, the third (wrongful) act can only occur when both the first and the second (wrongful) acts have occurred and so on. Any wrongful act results in an amount of damage to the injured party. The injuring parties are the tortfeasors who can be considered to be jointly liable for the full damage. The problem is how to apportion the full damage amongst the tortfeasors. In many real life situations, this problem is brought to court. This sharing problem is referred to as the Liability problem.

Historically, common law did not accept any apportionment among the tortfeasors, but evolution of common law in the 19th and 20th centuries led to the third Restatement of Torts (May 1999), providing basic principles and rules to apportion the damages. However, a systematic apportionment method is still the subject of research. In the existing literature on law and economics, it is a central topic to clarify whether or not a legal compensation scheme for liability problems is useful, see for instance Landes and Posner (1980), Shavell (1983), and Parisi and Singh (2010). These authors analyze the functioning of compensation schemes from the viewpoint of incentives. On the other hand, the viewpoint of fairness is also important. In fact, tort law prescribes an award of damages to achieve fair compensation for injury, see Boston (1995-1996). Although a few researchers have investigated the normative topic of liability problems (for instance, see Dehez and Ferey (2013) and Ferey and Dehez (2015)), this topic still needs further investigation.

In this paper, we analyze the functioning of compensation schemes from the viewpoint of fairness. For this purpose, we take an axiomatic approach. ${ }^{1}$ The axioms proposed in this paper are derived by taking into account tort law. Furthermore, we are interested in the situation where the determination of causal weights between the injuring parties is difficult in the following two senses. First, since a judge determines causal weights subjectively, fair compensation for injury may be difficult. Second, if the judge's transaction cost of this determination is high, then the determination may be impossible. Under this situation, we axiomatize the compensation scheme associated with the 'difference principle of social justice' à la Rawls (1971).

The liability problem as described in Dehez and Ferey (2013) has a linear structure in the sense that the agents are linearly ordered: the wrongful act of agent $i$ can only occur

\footnotetext{
${ }^{1}$ Axiomatic approaches to economic allocation problems, where the axioms are based on principles of distributive justice have been described in, e.g. Moulin (2003)
} 
when all agents $j<i$ behave wrongfully. In this paper, we consider the more general class of liability problems with rooted-tree structure. As an example, we consider the case where the injured party suffers an injury that is caused by four agents, agents 1, 2, 3, and 4 . Agent 1 has taken a wrongful act that is the root of the injury. After agent 1's wrongful act, agents 2 and 3 have taken wrongful acts. Without agent 1's wrongful act, agents 2 and 3's wrongful acts would not have occurred. On the other hand, agent 2's wrongful act does not affect agent 3's wrongful act and reversely. After agent's 1 wrongful act, the wrongful act of agent 3 might occur without the wrongful act of agent 2 and reversely, the wrongful act of agent 2 might occur without the wrongful act of agent 3 . Without the wrongful acts of both agents 1 and 2, agent 4's wrongful act would not have occurred. The situation underlying this example can be modeled by the rooted-tree graph of Figure 1 . In this graph, agent 1 is located at the root and has two branches, at one branch agent 1 is succeeded by agent 2 and agent 2 is succeeded by agent 4 , at the other branch agent 1 is succeeded by agent 3 . Agents 3 and 4 are the leafs of the tree. The rooted-tree represents the hierarchical structure of causation of the cumulative injury.

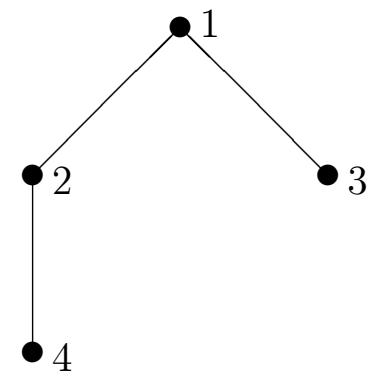

Figure 1: Rooted tree with two branches.

Formally, the liability problem is how to share the total damage amongst the $n$ tortfeasors, called the agents and indexed by $i=1, \ldots, n$. A compensation scheme is a sharing rule that determines for every liability problem the compensations that have to be paid by the individual tortfeasors. Note that a compensation scheme is a procedure (algorithm) that can be applied to every liability problem.

Following the existing literature, we use several notions of damages. Here, we explain the notions by using the case of liability problems with linear structure. Analogously to this case, it is easy to understand the notions of damages in the case of liability problems with rooted-tree structure. It is supposed that all damages can be measured monetary. Every agent $i$ has taken a wrongful act that causes an amount of damage $d_{i}$. However, the wrongful act of agent $i$ can only occur when all agents $1, \ldots, i-1$ have taken wrongful acts. So, if at least one of the wrongful acts of the agents $1, \ldots, i-1$ would not have occurred, 
then the wrongful act of agent $i$ would not have occurred. In the existing literature, see for instance Dehez and Ferey $(2013)^{2}$, the amount $d_{i}$ is called the direct damage resulting from the wrongful act of $i$. Furthermore, for every $i$ the cumulative damage up to $i$ is defined as $c_{i}=\sum_{k=1}^{i} d_{k}$ and the additional damage of $i$ is defined as $e_{i}=c_{n}-c_{i-1}=\sum_{k=i}^{n} d_{k}$. So, the cumulative damage up to agent $n$ is the total damage of the tortfeasors, while the additional damage of $i$ is the sum of all damages that would not have occurred without the wrongful act of $i$. Finally, we have the notion of potential damage. This type of damage is defined for every subset $S \subseteq\{1, \ldots, n\}$ of tortfeasors. Define $k(S)=0$ if agent 1 is not a member of $S$. Otherwise, define $k(S)$ as the highest indexed member of $S$ such that all consecutive agents $1, \ldots, k(S)$ also belong to $S$. Then the potential damage of $S$ is the cumulative damage $c_{k(S)}$, with $c_{0}=0$, i.e. it is sum of the damages that the members of $S$ cause when the members outside $S$ do not behave wrongfully.

Dehez and Ferey (2013) introduce a certain compensation scheme formalized by causal weights between the injuring parties and the list of additional damages. They show that for every liability problem this compensation scheme yields the (weighted) Shapley value of the corresponding transferable utility game (for short TU game) that assigns worth $v(S)=c_{k(S)}$ to every subset $S$ of tortfeasors, i.e., the worth of $S$ is the potential damage of $S$. The (weighted) Shapley value (Shapley 1953; Kalai and Samet 1987) is an established solution for TU games, and it is a game theoretic expression of fairness. Several notions of fairness underlying the Shapley value are proposed in the existing literature, for instance see Myerson (1980) and van den Brink (2001). In Ferey and Dehez (2015) a characterization of the compensation scheme yielding the Shapley value for liability problems is given.

As Dehez and Ferey (2013) point out, for a legal compensation scheme concerning tort law it should be required that for every liability problem it yields individual compensations that satisfy the following properties:

(i) Every injuring party should pay at least the potential damage that he would have caused alone.

(ii) Every injuring party should pay at most the additional damage that he would have caused. This principle is supported by the third Restatement of Torts, which is formulated by the American Law Institute.

For a liability problem with rooted-tree structure, based on these two properties we define in this paper two TU games, the lower-bound liability game and the upper-bound liability game. The first one assigns to every subset $S$ of tortfeasors a worth $v_{L}(S)$ that is equal to the potential damage of $S$. For problems with linear structure this game reduces to the liability game as defined in Dehez and Ferey (2013). The upper-bound liability game

\footnotetext{
${ }^{2}$ Here and in the sequel when we refer to Dehez and Ferey (2013), see also Ferey and Dehez (2015)
} 
assigns to every subset $S$ of tortfeasors a worth $v_{U}(S)$ that is equal to the additional damage of $S .^{3}$ It appears that these two games are the duals of each other in the game-theoretic sense.

A vector $x=\left(x_{1}, \ldots, x_{n}\right)$ of individual compensations, in the sequel shortly outcome, is in the core of the lower-bound game if for every $S$ it holds that $\sum_{i \in S} x_{i} \geq v_{L}(S)$, i.e., the total compensation to be paid by the members of $S$ is at least equal to their potential damage. Also, for every $S \subseteq N, x$ satisfies $\sum_{i \in S} x_{i} \geq v_{L}(S)$ if and only if $\sum_{i \in S} x_{i} \leq v_{U}(S)$, so an outcome in the core of the lower-bound liability game satisfies the Properties (i) and (ii) mentioned above. In fact, an outcome is in the core if and only if it satisfies this type of properties for every subset of tortfeasors. Generalizing a result of Dehez and Ferey (2013) for linear liability games, also for liability games with rooted tree structure the lower-bound liability game is convex.

In this paper, we propose three axioms and we show that these three axioms determine a unique compensation scheme that yields for every liability problem with rooted-tree structure an outcome in the core of the lower-bound liability game. As Property (i), the first axiom sets for every tortfeasor a uniform lower bound. This lower bound is the same for every tortfeasor, and for an individual tortfeasor the best possible outcome is an outcome in which he has to pay this lower bound. As Property (ii), the second axiom sets for every tortfeasor an individual upper bound. This upper bound differs over the tortfeasors and gives for every tortfeasor its worst possible outcome. In order to set the lower and upper bound axioms, per capita criteria are employed. This is because in liability problems it is often impossible to determine the causal weights between the injuring parties. In this situation, per capita criteria might be justified. The third and last axiom stems from the stylized fact that in the UK and America, a legal compensation scheme is based on so-called case system consistency, see for instance Ito (1978). This requires that the compensation scheme has the property that for every liability problem it provides an outcome that is consistent with the outcome that the same procedure generates for a different, but similar liability problem. In this paper, we propose leaf consistency. This type of case system consistency requires that for every liability problem with rooted-tree structure the compensation scheme is invariant when a leaf of the tree pays his compensation and leaves.

We show that the three axioms uniquely determine a compensation scheme. This scheme assigns to every liability problem with rooted-tree structure the so-called nucleolus of the lower bound liability game as outcome. The Nucleolus compensation scheme has two appealing properties.

First, the nucleolus (Schmeidler, 1969) is an established outcome for TU games. In fact, it is a game-theoretic expression of the 'difference principle of social justice' à la Rawls

\footnotetext{
${ }^{3}$ The additional damage of a subset $S$ of tortfeasors will be defined formally in the next section.
} 
(1971). So, when it is desirable that a legal compensation scheme for liability problems is to attain a Rawlsian outcome, the three axioms yield a useful compensation scheme.

Second, it is well known that the nucleolus of a game is in the core if the core is nonempty. Using the fact that the upper bound liability game is the dual of the lower bound liability game, it follows that the nucleolus of the lower bound liability game lexicographically maximizes the differences $v_{U}(S)-\sum_{i \in S} x_{i}$ of all subsets $S$ of tortfeasors, i.e. it makes the smallest difference over all subsets $S$ as high as possible, then the second smallest one, then the third smallest one, and so on. So, it maximizes (lexicographically) over all different subsets $S$ of tortfeasors the differences between the additional damage $v_{U}(S)$ and the actual compensation $\sum_{i \in S} x_{i}$ to be paid by the members of $S$. Loosely speaking, it minimizes (lexicographically) the dissatisfactions over the subsets of tortfeasors with respect to their 'worst-case' outcomes, i.e. the outcome in which $S$ has to pay the total amount $v_{U}(S)$ of its additional damage.

The remainder of the paper is organized as follows. In Section 2, preliminaries are given. In Section 3, the liability problem with rooted-tree structure and the corresponding liability games are given. In Section 4, we state and discuss the three axioms to be satisfied by a compensation scheme. In Section 5, we show that the compensation scheme that satisfies the axioms of Section 4 assigns to every liability problem the nucleolus of the corresponding lower-bound liability game. Section 6 contains concluding remarks, and discusses a comparison between the Shapley and nucleolus compensation schemes, and an incentive problem in the situation where the population of the tortfeasors is increasing.

\section{Preliminaries}

A cooperative game with transferable utility, or simply a TU game, is a pair $(N, v)$, where $N \subseteq \mathbb{N}$ is a finite set of players, and $v: 2^{N} \rightarrow \mathbb{R}$ is a characteristic function that assigns a worth $v(S) \in \mathbb{R}$ to every subset (usually called coalition) $S$ of $N$, satisfying $v(\emptyset)=0$. A TU game $(N, v)$ is convex if $v(S \cup T)+v(S \cap T) \geq v(S)+v(T)$ for all $S, T \subseteq N$. It is concave if these inequalities are reversed. We denote by $\mathcal{G}$ the class of all TU games. The subclass of all convex TU games is denoted by $\mathcal{G}^{\text {vex }}$ and the subclass of all concave TU games by $\mathcal{G}^{\text {cave }}$. For a game $(N, v) \in \mathcal{G}$, the dual game, denoted by $\left(N, v^{d}\right)$, assigns to every coalition $S$ what the 'grand coalition' $N$ loses if the players in $S$ stop cooperating, and thus is defined by $v^{d}(S)=v(N)-v(N \backslash S)$ for all $S \subseteq N$. Note that $v^{d}(\emptyset)=0$ and $v^{d}(N)=v(N)$. Further it holds that $(N, v) \in \mathcal{G}^{v e x}$ if and only if $\left(N, v^{d}\right) \in \mathcal{G}^{\text {cave }}$.

A payoff vector of TU game $(N, v)$ is a vector $x \in \mathbb{R}^{N}$ giving a payoff $x_{i} \in \mathbb{R}$ to every player $i \in N$. A payoff vector is efficient if $\sum_{i \in N} x_{i}=v(N)$. Given $(N, v) \in \mathcal{G}$, the 
preimputation set of $(N, v)$, denoted by $P I(N, v)$, is the set of all efficient payoff vectors. Further, the imputation set, denoted by $I(N, v)$, is the subset of all vectors in $P I(N, v)$ that satisfy $x_{i} \geq v(\{i\})$ for every $i \in N$ (individual rationality); the anti-imputation set, denoted by $A I(N, v)$, is the subset of all vectors in $P I(N, v)$ that satisfy $x_{i} \leq v(\{i\})$ for every $i \in N$. Note that these sets are not-empty if and only if $v(N) \geq \sum_{i \in N} v(\{i\})$, respectively $v(N) \leq \sum_{i \in N} v(\{i\})$. We denote by $\mathcal{G}^{I}$ the class of all TU-games with nonempty $I(N, v)$, and by $\mathcal{G}^{A I}$ the class of all TU-games with nonempty $A I(N, v)$. Note that $\mathcal{G}^{\text {vex }}$ is a subset of $\mathcal{G}^{I}$ and $\mathcal{G}^{\text {cave }}$ is a subset of $\mathcal{G}^{A I}$.

The core of a game $(N, v)$, denoted by $C(N, v)$, is the set of (efficient) payoff vectors that are group stable, and is given by

$$
C(N, v)=\left\{x \in P I(N, v) \mid \sum_{i \in S} x_{i} \geq v(S) \text { for all } S \subseteq N\right\} .
$$

Note that $C(N, v)$ is a subset of $I(N, v)$ and that it might be empty. Every game $(N, v) \in$ $\mathcal{G}^{\text {vex }}$ has a non-empty core. A vector $x \in C(N, v)$ satisfies the requirement that for every coalition $S$ the total payoff is at least equal to its own worth. This is reasonable when $(N, v)$ is a profit game, i.e., the worth $v(S)$ is the total revenue that the members of $S$ can achieve by cooperating. However, when $v$ is a cost game, i.e., coalition $S$ has costs $v(S)$ when it stands alone, then the worth should be considered as upper bounds on the contributions. For a cost game it makes sense to apply the anti-core of a game $(N, v)$, denoted by $A C(N, v)$. This set of efficient payoff vectors is given by

$$
A C(N, v)=\left\{x \in P I(N, v) \mid \sum_{i \in S} x_{i} \leq v(S) \text { for all } S \subseteq N\right\} .
$$

The anti-core $A C(N, v)$ is a subset of $A I(N, v)$ and might be empty, but every game $(N, v) \in \mathcal{G}^{\text {cave }}$ has a non-empty anti-core.

For a given subset $\mathcal{G}^{\prime}$ of the class $\mathcal{G}$ of all TU-games, a (single-valued) solution is a function $f$ that assigns to every game $(N, v)$ in $\mathcal{G}^{\prime}$ a payoff vector $f(N, v) \in P I(N, v)$. Note that in this paper we require that a solution assigns to each game an efficient payoff vector. The best-known solution on the class $\mathcal{G}$ of all TU-games is the Shapley value (Shapley, 1953), denoted $S h$. This solution assigns to every game $(N, v) \in \mathcal{G}$ the payoff vector $\operatorname{Sh}(N, v)$ given by ${ }^{4}$

$$
S h_{i}(N, v)=\sum_{S \subseteq N: i \in S} \frac{(|N|-|S|) !(|S|-1) !}{|N| !}(v(S)-v(S \backslash\{i\})) \text { for all } i \in N .
$$

So, for every player $i \in N$ the payoff is a weighted sum of its marginal contributions $v(S)-v(S \backslash\{i\})$ to the coalitions $S$ containing $i$. When $(N, v)$ is convex, then $S h(N, v) \in$

\footnotetext{
${ }^{4}$ For a finite set $A$, we denote by $|A|$ the number of elements in $A$ (cardinality of $A$ ).
} 
$C(N, v)$. However, in general, on the domain of TU games with non-empty cores it might be that the Shapley value is not in the core. Further it holds that the Shapley value is self-dual (see Kalai and Samet (1987)), saying that for every $(N, v) \in \mathcal{G}$ it holds that $\operatorname{Sh}\left(N, v^{d}\right)=\operatorname{Sh}(N, v) .^{5}$

Another well-known solution on the class of all TU-games is the prenucleolus. Given a TU game $(N, v) \in \mathcal{G}$, we define for payoff vector $x \in P I(N, v)$ and coalition $S \subseteq N$ the excess of $S$ with respect to $x$ as

$$
e(S, x, v) \equiv v(S)-\sum_{i \in S} x_{i}
$$

When the payoffs are revenues, i.e., the payoffs are payments to the players, the excess $e(S, x, v)$ can be seen as a measure of dissatisfaction of coalition $S$. The bigger the excess of $S$ and thus the bigger the difference between its own worth $v(S)$ and its payoff $\sum_{i \in S} x_{i}$ received by the members of $S$, the more dissatisfied coalition $S$ is. Now, let $\theta(x, v) \in \mathbb{R}^{2^{N}}$ be the vector obtained by arranging all the excesses in non-increasing order, so the first component of $\theta(x, v)$ is the excess of a coalition with the highest excess, the second component is the excess of a coalition with the highest excess under the remaining coalitions, and so on. Then the prenucleolus is the solution on $\mathcal{G}$ that assigns to every game $(N, v) \in \mathcal{G}$ the unique vector in $P I(N, v)$ that minimizes lexicographically the dissatisfactions. To be precise, the prenucleolus assigns to every game $(N, v)$ the unique payoff vector $x \in P I(N, v)$ such that for every $y \in P I(N, v) \backslash\{x\}$ there exist a component $k$ such that $\theta_{h}(x, v)=\theta_{h}(y, v)$ for all $h<k$, and $\theta_{k}(x, v)<\theta_{k}(y, v)$, i.e. the prenucleolus assigns to $(N, v) \in \mathcal{G}$ the unique payoff vector $x \in P I(N, v)$, such that for every other vector $y \in P I(N, v)$ there exists a number $k$ such that the $k-1$ biggest excesses of $x$ and $y$ are equal and the next biggest excess of $x$ is smaller than the next biggest excess of $y$. We denote the prenucleolus of a game $(N, v)$ by $P N u c(N, v)$.

The nucleolus is a solution defined for every game with $I(N, v)$ not-empty, so on the subclass of games $\mathcal{G}^{I}$. It assigns to every $(N, v) \in \mathcal{G}^{I}$ the unique individually rational payoff vector $x \in I(N, v)$ that minimizes lexicographically the dissatisfactions over all vectors in $I(N, v)$. We denote the nucleolus of a game $(N, v)$ by $\operatorname{Nuc}(N, v)$. When the core is nonempty, $\operatorname{Nuc}(N, v) \in C(N, v)$. Further, on the class of games with non-empty $C(N, v)$ the nucleolus coincides with the prenucleolus (Schmeidler, 1969). In particular it holds that $\operatorname{Nuc}(N, v)=P \operatorname{Nuc}(N, v)$ when $(N, v)$ is convex.

Similarly, for a game $(N, v)$ we define the anti-prenucleolus, denoted $A P N u c(N, v)$, and for a game $(N, v)$ in the subclass $\mathcal{G}^{A I}$ the anti-nucleolus, denoted $A N u c(N, v)$. The antiprenucleolus is the unique payoff vector $x \in P I(N, v)$ such that $-\theta(v, x)$ is lexicographically

\footnotetext{
${ }^{5}$ The notion of (self-)duality plays an important role in axiomatizing solutions for TU games. For instance, see Oishi, Nakayama, Hokari, and Funaki (2016).
} 
smaller than $-\theta(v, y)$ for every $y \in P I(N, v)$, i.e., it is the unique vector in $P I(N, v)$ that minimizes lexicographically the vector of negative excesses $\sum_{i \in S} x_{i}-v(S)$ for all $S \subseteq N$. So, for a cost game, the anti-prenucleolus lexicographically maximizes the cost savings $v(S)-\sum_{i \in S} x_{i}$ for all $S \subseteq N$. The anti-nucleolus assigns to every $(N, v) \in \mathcal{G}^{A I}$ the unique payoff vector $x \in I(N, v)$ that minimizes lexicographically the vector of negative excesses over all vectors in $A I(N, v)$. When the anti-core is non-empty, $\operatorname{ANuc}(N, v) \in A C(N, v)$. When $(N, v) \in \mathcal{G}^{\text {cave }}$ it holds that $\operatorname{ANuc}(N, v)=A P N u c(N, v)$.

In the next sections the following proposition, which follows from Oishi and Nakayama (2009), will appear to be useful. Recall that $(N, v) \in \mathcal{G}^{v e x}$ if and only if $\left(N, v^{d}\right) \in \mathcal{G}^{\text {cave }}$.

Proposition 2.1 For every $(N, v) \in \mathcal{G}^{v e x}$ it holds that

(i) $A C\left(N, v^{d}\right)=C(N, v)$,

(ii) $\operatorname{ANuc}\left(N, v^{d}\right)=\operatorname{Nuc}(N, v)$.

We now introduce rooted trees. First, a directed graph or digraph is a pair $(N, D)$, where $N$ is a set of nodes and the collection of ordered pairs $D \subseteq\{(i, j) \mid i, j \in N, i \neq j\}$ is a set of arcs. In this paper, the nodes represent the players in a game, and therefore we refer to the nodes as players. We denote the set of all digraphs by $\mathcal{D}$. For $(N, D) \in \mathcal{D}$, a sequence of $k$ different players $\left(i_{1}, \ldots, i_{k}\right)$ is a (directed) path if $\left(i_{l}, i_{l+1}\right) \in D$ for $l=1, \ldots, k-1$. For $i \in N$, a player $j \in N$ is a subordinate of $i$ if there is a path $\left(i_{1}, \ldots, i_{k}\right)$ with $i_{1}=i$ and $i_{k}=j$. Player $i$ is a superior of $j$ if and only if $j$ is a subordinate of $i$. We denote $F_{D}(i)$ as the set of subordinates of $i$ and $P_{D}(i)$ as the set of superiors of $i$ in $(N, D)$. We also denote $F_{D}^{0}(i)=F_{D}(i) \cup\{i\}$ and $P_{D}^{0}(i)=P_{D}(i) \cup\{i\}$. Also for all $S \subseteq N$ we define $F_{D}(S)=\cup_{i \in S} F_{D}(i)$ and similarly $P_{D}(S)=\cup_{i \in S} P_{D}(i), F_{D}^{0}(S)=\cup_{i \in S} F_{D}^{0}(i)$ and $P_{D}^{0}(S)=\cup_{i \in S} P_{D}^{0}(i)$.

A node $i \in N$ is called a top player in $(N, D)$ if $P_{D}(i)=\emptyset$. A digraph $(N, D)$ is a (directed) rooted tree with root $i$ when (i) player $i$ is the unique top player and (ii) for all $j \neq i$ there is a unique path from $i$ to $j$. In the sequel we denote by $\mathcal{D}_{t}$ the class of rooted trees and an element of $\mathcal{D}_{t}$ by $(N, T)$. Note that for a rooted tree $(N, T)$ with root $i$ it holds that $F_{T}(i)=N \backslash\{i\}$ and for every node $j \neq i$ there is precisely one player $k \in P_{T}(j)$ such that $(k, j) \in T$. This player is called the predecessor of $j$ and denoted by $p(j)$. A player is called a leaf of $(N, T)$ if $F_{T}(i)=\emptyset$. We denote the set of all leafs by $L(T)$. We say that a tree $(N, T)$ is linear if $|L(T)|=1$. In this case it holds that for every player $k$ not in $L(T)$ there is precisely one $h$ such that $(k, h) \in T$. For a tree $(N, T)$ with root $i$, let $M \subseteq N$ be such that (i) $|M| \geq 2$, (ii) $i \in M$, (iii) for every $j \in M \backslash\{i\}$ all nodes on the (unique) directed path from $i$ to $j$ are also in $M$. Then $(M, T(M))$ denotes the subtree of $(N, T)$ restricted to $M$ and $\mathcal{T}$ denotes the collection of all subtrees $(M, T(M))$ of $(N, T)$. 
A permission tree game is a triple $(N, v, T)$ with $N \subset \mathbb{N}$ a finite set of players, $(N, v) \in \mathcal{G}$ a TU-game and $(N, T) \in \mathcal{D}_{t}$ a rooted tree on $N$. In those games, it is assumed that the tree represents a hierarchy that imposes restrictions on the forming of coalitions. Solutions for permission tree games have been discussed in for instance van den Brink, Herings, van der Laan and Talman (2016) and van den Brink, Dietz, van der Laan and Xu (2015). One of these solutions is the permission value, based on the so-called conjunctive approach to permission structures as developed in Gilles, Owen and van den Brink (1992). In this approach, it is assumed that a coalition is feasible if and only if for every player in the coalition all its predecessors are also in the coalition. feasible coalitions is given by

$$
\Phi_{T}=\left\{S \subseteq N \mid P_{T}(i) \subseteq S \text { for all } i \in S\right\}
$$

In this paper we only consider triples $(N, v, T)$ with the permission structure $(N, T) \in \mathcal{D}_{t}$ a rooted tree. We denote by $\mathcal{G}_{\mathcal{T}}$ the collection of all permission tree games. A (single-valued) solution $f$ on $\mathcal{G}_{\mathcal{T}}$ assigns a unique payoff vector $f(N, v, T) \in \mathbb{R}^{N}$ to every $(N, v, T) \in \mathcal{G}_{\mathcal{T}}$. For $S \subseteq N$, let $\sigma_{T}(S)=\bigcup_{R \in \Phi_{T}: R \subseteq S} R$ be the largest feasible subset ${ }^{6}$ of $S$. Following Gilles Owen and van den Brink (1992), the induced permission restricted game of $(N, v, T)$ is the game $\left(N, r_{N, v, T}\right) \in \mathcal{G}$ given by

$$
r_{N, v, T}(S)=v\left(\sigma_{T}(S)\right) \text { for all } S \subseteq N
$$

and the permission value $\psi$ on $\mathcal{G}_{\mathcal{T}}$ is the solution that assigns to every $(N, v, T) \in \mathcal{G}_{T}$ the Shapley value of the associated permission restricted game, thus

$$
\psi(N, v, T)=S h\left(N, r_{N, v, T}\right) \text { for all }(N, v, T) \in \mathcal{G}_{\mathcal{T}}
$$

\section{$3 \quad$ Liability problems with rooted-tree structure}

A liability problem with rooted-tree structure (shortly a liability problem) is a triple $(N, T, d)$ with $(N, T) \in \mathcal{D}_{t}$ a rooted tree on $N$ and $d \in \mathbb{R}_{+}^{N}$ a profile of non-negative damages, with $d_{i}$ the (direct) damage caused by tortfeasor $i \in N$. We denote the class of all liability problems by $\mathcal{L}$. We also define the following notions.

Total damage For $S \subseteq N$, the total damage of $S$ is $d_{S}=\sum_{j \in S} d_{j}$.

Cumulative damage For $S \subseteq N$, the cumulative damage up to $S$ is $c_{S}=\sum_{j \in P_{T}^{0}(S)} d_{j}$. Additional damage For $S \subseteq N$, the additional damage of $S$ is $e_{S}=\sum_{j \in F_{T}^{0}(S)} d_{j}$. Potential damage For every subset $S \subseteq N$, the potential damage of $S$ is $b_{S}=\sum_{j \in S: P_{T}(j) \subseteq S} d_{j}$.

\footnotetext{
${ }^{6}$ Every coalition having a unique largest feasible subset follows from the fact that $\Phi_{T}$ is union closed, i.e. for every $E, F \in \Phi_{T}$ it holds that $E \cup F \in \Phi_{T}$
} 
The total damage of $S$ is the sum of the damages of the players in $S$. The cumulative damage of $S$ is the sum of the damages of the players in $S$ and all their superiors. The additional damage of $S$ is the sum of the damages of the players in $S$ and all their subordinates and can be seen as the sum of all damages that would have been avoided when none of the members of $S$ exercised a wrongful act. The potential damage of $S$ is the sum of all damages that the members of $S$ cause when the members outside $S$ do not behave wrongfully. In case the tree is linear and $S=\{j\}$ for some $j \in N$, these notions coincide with the notions of Dehez and Ferey (2013) as discussed in the Introduction. For ease of notation we denote $d_{S}=d_{i}$ if $S=\{i\}$ and similarly for the other notions. Note that $d_{N}=c_{N}=e_{N}=b_{N}$, and for the root $i, d_{i}=c_{i}=b_{i}$ and $e_{i}=\sum_{j \in F_{T}^{0}(i)} d_{j}=d_{N}$. The following example illustrates the different notions of damages mentioned above.

Example 3.1 Consider six players and rooted-tree $(N, T)$ with $N=\{1,2,3,4,5,6\}$ and $T=\{(1,2),(2,3),(2,4),(1,5),(5,6)\}$, see Figure 2. Table 1 gives the four notions of damages for two different sets $S$. Note that $d_{N}=\sum_{i \in N} d_{i}$.

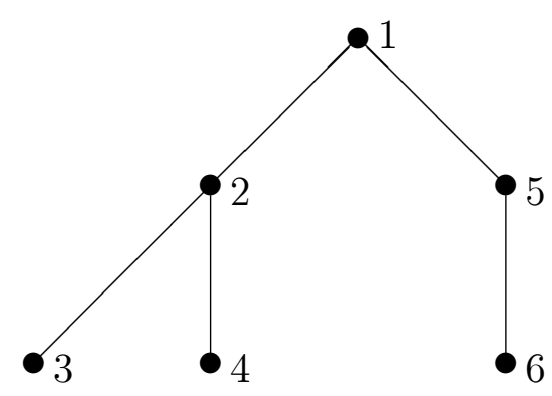

Figure 2: Rooted tree with six players. 
Table 1. Four notions of damages for the tree of Figure 2.

\begin{tabular}{|l||l|l|}
\hline & \multicolumn{1}{|c|}{$S=\{1,4,6\}$} & \multicolumn{1}{|c|}{$S=\{2,6\}$} \\
\hline Total damage $d_{S}$ & $d_{1}+d_{4}+d_{6}$ & $d_{2}+d_{6}$ \\
\hline Cumulative damage $c_{S}$ & $d_{N}-d_{3}$ & $d_{1}+d_{2}+d_{5}+d_{6}$ \\
\hline \hline Additional damage $e_{S}$ & $d_{N}$ & $d_{2}+d_{3}+d_{4}+d_{6}$ \\
\hline Potential damage $b_{S}$ & $d_{1}$ & 0 \\
\hline
\end{tabular}

Given $(N, T, d) \in \mathcal{L}$, an allocation for $(N, T, d)$ is a non-negative vector $x \in \mathbb{R}_{+}^{N}$ such that $\sum_{i \in N} x_{i}=d_{N}$. A compensation scheme for liability problems is a mapping $\varphi$ on $\mathcal{L}$ that associates with every problem $(N, T, d) \in \mathcal{L}$ an allocation $\varphi(N, T, d) \in \mathbb{R}_{+}^{N}$.

We generalize the liability game as defined by Dehez and Ferey (2013) for liability problems with linear structure to the class of liability problems with rooted tree structure. For liability problem $(N, T, d) \in \mathcal{L}$ the corresponding lower-bound liability game is the game $\left(N, v_{L}\right)$ where the worth of a coalition $S$ is its potential damage, i.e. for all $S \subseteq N$,

$$
v_{L}(S)=b_{S}
$$

Dehez and Ferey (2013) focus on the Shapley value of the (lower-bound) liability game. In the sequel we define the Shapley value of a liability problem $(N, T, d)$ as the Shapley value of the corresponding lower-bound liability game. Formally we have the following definition.

Definition 3.2 The Shapley compensation scheme is the mapping Sh on $\mathcal{L}$ that associates with every problem $(N, T, d) \in \mathcal{L}$ the Shapley value of its corresponding lower-bound liability game $\left(N, v_{L}\right)$ :

$$
\operatorname{Sh}(N, T, d) \equiv \operatorname{Sh}\left(N, v_{L}\right) .
$$

In the literature on game theory, the lower-bound liability game is known as the peergroup game associated to peer-group situation $(N, T, d)$, see e.g. Brânzei, Fragnelli and Tijs (2002). A peer-group game, and so the game $\left(N, v_{L}\right)$, is convex. Further it holds that $\left(N, v_{L}\right)$ is the permission restricted game $\left(N, r_{N, v, T}\right)$ of the additive game $(N, v)$ with characteristic function defined as $v(S)=d_{S}$ for all $S \subseteq N$. Therefore, the Shapley value of game $\left(N, v_{L}\right)$ is equal to the permission value of the permission tree game $(N, T, v)$. Since $\left(N, v_{L}\right)$ is convex, its Shapley value is in its core: $\operatorname{Sh}\left(N, v_{L}\right) \in C\left(N, v_{L}\right)$. It follows that $\sum_{i \in S} S h_{i}(N, T, d)=\sum_{i \in S} S h_{i}\left(N, v_{L}\right) \geq v_{L}(S)$ for every $S \subseteq N$ and thus the Shapley compensation scheme satisfies the requirement that for every $S \subseteq N$ the total compensation paid by its members is at least equal to $v_{L}(S)$, being the potential damage of $S$. The 
Shapley compensation scheme thus satisfies Property (i) of the Introduction as required in Dehez and Ferey (2013).

While Dehez and Ferey (2013) focus on the Shapley value of the (lower-bound) liability game, in this paper we consider the nucleolus. We define the nucleolus compensation scheme as the mapping that assigns to every liability problem $(N, T, d)$ the nucleolus of its corresponding lower-bound liability game $\left(N, v_{L}\right)$.

Definition 3.3 The nucleolus compensation scheme is the mapping Nuc on $\mathcal{L}$ that associates with every problem $(N, T, d) \in \mathcal{L}$ the nucleolus of its corresponding lower-bound liability game $\left(N, v_{L}\right)$ :

$$
\operatorname{Nuc}(N, T, d) \equiv N u c\left(N, v_{L}\right)
$$

Since the nucleolus of a game is in its core (if the latter is non-empty), the $\mathrm{Nu}$ cleolus compensation scheme satisfies the requirement that for every $S \subseteq N$ the total compensation paid by its members is at least equal to the potential damage of $S$ $\left(\sum_{i \in S} N u c_{i}(N, T, d)=\sum_{i \in S} N u c_{i}\left(N, v_{L}\right) \geq v_{L}(S)\right.$ for every $\left.S \subseteq N\right)$ and thus respects Property (i) of the Introduction. Nevertheless, we now run into a difficulty about the interpretation of the core and nucleolus. Typically it is considered to be desirable that a payoff vector is in the core of the game. This is called core-stability, saying that every coalition $S$ gets at least its own worth $v(S)$ and so the members of $S$ don't have an incentive to deviate from the grand coalition $N$. However, this holds for profit games in which $v(S)$ is the worth that the members of $S$ can earn by themselves without cooperating with the others, and the entries of $x$ yield payoffs that are paid to the players. In contrast to this usual situation, the Shapley value or the nucleolus of a liability game gives a vector of compensations to be paid by the players in the game, i.e., by the tortfeasors. They are not looking for core stability, on the contrary they want to pay as little as possible.

Therefore, the lower-bound liability game should not be considered as a game that is played by the tortfeasors themselves, but as a model to help the court to determine the compensations to be paid to the injured party. With this interpretation the game gives for every subset (coalition) of tortfeasors, the lower-bounds of their compensations and for instance the Shapley value or the nucleolus can be applied to determine how much every coalition has to pay in addition to its lower bound $v_{L}(S)$. According to the Shapley value, these additional payments are determined by the marginal contributions of the players. ${ }^{7}$ In contrast, the nucleolus is determined by the excesses of the coalitions. However, for a liability game $\left(N, v_{L}\right)$ the excess $e\left(S, x, v_{L}\right)=v_{L}(S)-\sum_{i \in S} x_{i}$ is now a measure of

\footnotetext{
${ }^{7}$ Since the marginal contributions of a player do not depend on the damages of his superiors in the tree, this also implies that the Shapley value satisfies the property that the compensation to be paid by a tortfeasor does not depend on the damages of its superiors, see Ferey and Dehez (2015).
} 
satisfaction of $S$ at $x$, because the bigger the excess is, the lower the total amount of compensation that the members of $S$ have to pay. So, while in a game in which the payoff vector yields payments to the players the nucleolus minimizes lexicographically the vector of dissatisfactions, in the liability game the nucleolus minimizes lexicographically the vector of satisfactions. This is counterintuitive. Even if we consider the game as a model used by the court to determine the compensations, there is no a priori reason to do so.

Nevertheless, the Nucleolus compensation scheme is justified as a reasonable solution when we consider the second requirement of Dehez and Ferey (2013), namely that every tortfeasor should pay at most the additional damage that he would have caused, see Property (ii) in the Introduction. This principle is supported by the third Restatement of Torts as formulated by the American Law Institute. Based on this property, we define for a liability problem $(N, T, d) \in \mathcal{L}$ the corresponding upper-bound liability game as the game $\left(N, v_{U}\right)$ defined by setting, for all $S \subseteq N$,

$$
v_{U}(S)=e_{S},
$$

i.e. the worth of a coalition $S$ is the additional damage that the agents in $S$ might cause. ${ }^{8}$ The next lemma states that $\left(N, v_{U}\right)$ is the dual game of $\left(N, v_{L}\right)$. Because $\left(N, v_{L}\right)$ is convex this implies that $\left(N, v_{U}\right)$ is concave.

Lemma 3.4 For a liability problem $(N, T, d) \in \mathcal{L}$, the upper-bound liability game $\left(N, v_{U}\right)$ is the dual of the lower-bound liability game $\left(N, v_{L}\right)$.

Proof. For $S \subseteq N$, we have that $v_{L}(S)=b_{S}=\sum_{j \in S: P_{T}(j) \subseteq S} d_{j}$. Now, note that

$$
\left\{j \in S: P_{T}(j) \subseteq S\right\}=S \backslash F_{T}(N \backslash S)
$$

i.e., for every $S \subseteq N$, the set of players in $S$ such that all their predecessors are also in $S$ coincides with the set of players in $S$ that are not subordinates of the players in $N \backslash S$. With $F_{T}^{0}(N \backslash S)=F_{T}(N \backslash S) \cup(N \backslash S)$ it follows that $S \backslash F_{T}(N \backslash S)=N \backslash F_{T}^{0}(N \backslash S)$. Hence for every $S \subseteq N$ we obtain that $v_{L}(S)=\sum_{j \in N: j \notin F_{T}^{0}(N \backslash S)} d_{j}$ and thus

$$
\begin{gathered}
v_{L}^{d}(S)=v_{L}(N)-v_{L}(N \backslash S)=\sum_{j \in N} d_{j}-\sum_{j \in N: j \notin F_{T}^{0}(N \backslash(N \backslash S))} d_{j}= \\
\sum_{j \in F_{T}^{0}(N \backslash(N \backslash S))} d_{j}=\sum_{j \in F_{T}^{0}(S)} d_{j}=e_{S}=v_{U}(S) .
\end{gathered}
$$

\footnotetext{
${ }^{8}$ For a liability problem with linear structure the game $\left(N, v_{U}\right)$ is a so-called airport game, see Littlechild and Owen (1973).
} 
We now have the following proposition, where statement (i) follows because the Shapley value is self-dual and the other two statements follow from applying Proposition 2.1 to the convex game $\left(N, v_{L}\right)$ and its dual $\left(N, v_{U}\right)$.

Proposition 3.5 For every liability $\operatorname{problem}(N, T, d) \in \mathcal{L}$ the corresponding games $\left(N, v_{L}\right)$ and $\left(N, v_{U}\right)$ satisfy the following statements:

(i) $\operatorname{Sh}\left(N, v_{L}\right)=\operatorname{Sh}\left(N, v_{U}\right)$,

(ii) $C\left(N, v_{L}\right)=A C\left(N, v_{U}\right)$,

(iii) $\operatorname{Nuc}\left(N, v_{L}\right)=A N u c\left(N, v_{U}\right)$.

Statement (i) implies that the Shapley value of the lower-bound liability game is equal to the Shapley value of the upper-bound liability game. Since $\left(N, v_{L}\right)$ convex and thus $\operatorname{Sh}\left(N, v_{L}\right) \in C\left(N, v_{L}\right)$, statement (ii) implies that $\operatorname{Sh}\left(N, v_{L}\right) \in A C\left(N, v_{U}\right)$, and thus $S h\left(N, v_{L}\right)$ satisfies for every coalition $S$ the upper-bound requirement that $\sum_{i \in S} S h_{i}\left(N, v_{L}\right) \leq$ $e_{S}$. This also holds for $N u c\left(N, v_{L}\right)$. From statement (iii) it follows that $N u c\left(N, v_{L}\right)$ lexicographically maximizes the cost savings $v_{U}(S)-\sum_{i \in S} x_{i}$ with respect to the upper-bound liability game. So, when the court decides to implement $\operatorname{Nuc}\left(N, v_{L}\right)=\operatorname{ANuc}\left(N, v_{U}\right)$, the smallest cost saving over all coalitions $S$ is made as large as possible, then the second smallest is made as large as possible, then the third smallest, and so on. This is a very desirable property and gives a strong motivation for the use of the nucleolus.

We now consider the computation of the compensations for both the Shapley value and the nucleolus. The Shapley value is easy to compute for liability games. Recall that $\left(N, v_{L}\right)$ is the peer-group game associated to the peer-group situation $(N, T, d)$ and also that it is the permission restricted game $\left(N, r_{N, v, T}\right)$ of the additive game $(N, v)$ with its characteristic function defined as $v(S)=d_{S}$ for all $S \subseteq N$. For these games, it is well-known that the Shapley value distributes the damage $d_{i}$ of a player $i \in N$ equally amongst player $i$ and all its superiors in $(N, T)$. This gives the following expression for the compensation to be paid by a tortfeasor $j \in N$ according to the Shapley compensation scheme:

$$
S h_{j}(N, T, d)=\sum_{i \in F_{T}^{0}(j)} \frac{d_{i}}{\left|P_{T}^{0}(i)\right|} .
$$

So, when applying the Shapley value the compensation to be paid by tortfeasor $j$ is the sum of all his shares in the damages of himself and his subordinates.

There is no explicit formula available for the nucleolus, but the nucleolus compensations can be computed by the algorithm given in Brânzei, Solymosi and Tijs (2005) for peergroup games. Given a liability problem $(N, T, d) \in \mathcal{L}$, we assume without loss of generality 
that $1 \in N$ is the root of $(N, T)$. For a subtree $(M, T(M)) \in \mathcal{T}$ (see Section 2), let $(M, T(M), a), a \in \mathbb{R}_{+}^{M}$ be a liability problem on $M$. We define for every $j \in M \backslash\{1\}$,

$$
\tau_{j}(M, T(M), a) \equiv \frac{\sum_{k \in F_{T(M)}^{0}(j)} a_{k}}{\left|F_{T(M)}^{0}(j)\right|+1} .
$$

The Nucleolus compensation scheme is obtained by the following algorithm, where $x=$ $\operatorname{Nuc}(N, T, d) \equiv \operatorname{Nuc}\left(N, v_{L}\right)$. Recall that $p(j)$ is the predecessor of $j$ in $(N, T)$ and so also in every subtree $(M, T(M))$ containing $j$.

\section{Nucleolus algorithm:}

Step 0: Set $M=N$ and $a=d$.

Step 1: Find a $j \in M \backslash\{1\}$ such that $\tau_{j}(M, T(M), a)=\min _{m \in M \backslash\{1\}} \tau_{m}(M, T(M), a)$.

Step 2: For every $k \in F_{T(M)}^{0}(j)$, set $x_{k}=\tau_{j}(M, T(M), a)$. If $\left|M \backslash F_{T(M)}^{0}(j)\right| \geq 2$, go to Step 3. Otherwise, set $x_{1}=d_{N}-\sum_{k \in N \backslash\{1\}} x_{k}$ and stop.

Step 3: Set $M \equiv M \backslash F_{T(M)}^{0}(j)$ and set $a_{p(j)} \equiv a_{p(j)}+x_{j}$. Return to Step 1 .

Note that in Step 2, if $\left|M \backslash F_{T(M)}^{0}(j)\right|=1$, then $M \backslash F_{T(M)}^{0}(j)=\{1\}$. In the next Example we illustrate the above procedure.

Example 3.6 Let $(N, T, d)$ be the liability problem with rooted tree as given in Figure 2 and with vector of damages given by $d=(0,12,40,36,12,30)$.

Using formula (3.1) we have the following computations for the Shapley compensation scheme, starting with the leafs: $S_{3}(N, T, d)=\frac{d_{3}}{3}=\frac{40}{3}, S h_{4}(N, T, d)=\frac{d_{4}}{3}=12$, $S h_{6}(N, T, d)=\frac{d_{6}}{3}=10$. Next we obtain $S h_{2}(N, T, d)=\frac{d_{2}}{2}+S h_{3}(N, T, d)+S h_{4}(N, T, d)=$ $6+12+\frac{40}{3}=\frac{94}{3}, S h_{5}(N, T, d)=\frac{d_{5}}{2}+S h_{6}(N, T, d)=6+10=16$ and finally $S h_{1}(N, T, d)=$ $d_{1}+S_{2}(N, T, d)+S h_{5}(N, T, d)=0+\frac{94}{3}+16=\frac{142}{3}$. Thus $\operatorname{Sh}(N, T, d)=\left(\frac{142}{3}, \frac{94}{3}, \frac{40}{3}, 12,16,10\right)$.

To apply the algorithm for computing the nucleolus payoffs, let $x=\operatorname{Nuc}(N, T, d)$. Then the algorithm performs as follows.

Step 0: Set $M=N$ and $a=d$.

Iteration 1:

Step 1: $\min \left\{\frac{30}{2}, \frac{12+30}{3}, \frac{36}{2}, \frac{40}{2}, \frac{12+40+36}{4}\right\}=14=\tau_{5}(M, T(M), a)$.

Step 2: $x_{5}=x_{6}=14$.

Step 3: Set $M=\{1,2,3,4\}$ and $a=\left(d_{1}+14, d_{2}, d_{3}, d_{4}\right)=(14,12,40,36)$.

Iteration 2:

Step 1: $\min \left\{\frac{36}{2}, \frac{40}{2}, \frac{12+40+36}{4}\right\}=18=\tau_{4}(M, T(M), a)$. 
Step 2: $x_{4}=18$.

Step 3: Set $M=\{1,2,3\}$ and $a=\left(d_{1}+14, d_{2}+18, d_{3}\right)=(14,30,40)$.

Iteration 3:

Step 1: $\min \left\{\frac{40}{2}, \frac{30+40}{3}\right\}=20=\tau_{3}(M, T(M), a)$.

Step 2: $x_{3}=20$.

Step 3: Set $M=\{1,2\}$ and $a=\left(d_{1}+14, d_{2}+18+20\right)=(14,50)$.

Iteration 4:

Step 1: $\tau_{2}(M, T(M), a)=\frac{50}{2}=25$.

Step 2: $x_{2}=25$ and $x_{1}=d_{N}-\sum_{k \in N \backslash\{1\}} x_{k}=39$. Stop.

We have found that $\operatorname{Nuc}(N, T, d)=x=(39,25,20,18,14,14)$.

From formula (3.1) it follows immediately that the Shapley compensation scheme satisfies monotonicity in the sense that for every player $j \in N \backslash L(T)$ it holds that $j$ has to pay at least as much as any of his subordinates (and strictly more when $d_{j}>0$ ). This property also holds for the nucleolus compensation scheme, albeit that compensations can be equal when $d_{j}>0$. We call this weak monotonicity. ${ }^{9}$

\section{Lemma 3.7 Weak Monotonicity}

The compensation scheme $N u$ on the class $\mathcal{L}$ of liability problems satisfies that for every $(N, T, d) \in \mathcal{L}$ it holds for every $j \in N \backslash L(T)$ that

$$
N u c_{j}(N, T, d) \geq N u c_{k}(N, T, d) \text { for every } k \in F_{T}(j) \text {. }
$$

Proof. The proof follows from the algorithm. In the first iteration the algorithm starts with $(N, T, d)$. Let $k \in N \backslash\{1\}$ be the player such that $\tau_{k}(N, T, d)=\min _{j \in N \backslash\{1\}} \tau_{j}(N, T, d)$. Then $N u c_{h}(N, T, d)=\tau_{k}(N, T, d)$ for every $h \in F_{T}^{0}(k)$ and so the property holds for $k$ and all his subordinates. In the second iteration we have $M=N \backslash F_{T}^{0}(k)$ and the reduced liability problem $(M, T(M), a)$ with $a_{p(k)}=d_{p(k)}+\tau_{k}(N, T, d)$, and $a_{h}=d_{h}$ for every $h \in M \backslash\{p(k)\}$. We show that the minimal $\tau_{j}(M, T(M), a)$ in the second iteration is at least equal to $\tau_{k}(N, T, d)$. For simplicity of notation, denote $A_{j}=\tau_{j}(N, T, d)$ and $n_{j}=\left|F_{T}^{0}(j)\right|$ for every $j \in N \backslash\{1\}$.

First, consider a player $h \in M$ that is neither a subordinate of $k$ in $(N, T, d)$, nor a superior of $k$ in $(N, T, d)$. Then $\tau_{h}(M, T(M), a)=\tau_{h}(N, T, d)=A_{h} \geq A_{k}$ (since $A_{k}$ was minimal in the previous iteration). Second, consider a superior $h$ of $k$ in $(N, T, d)$. Now note that in the first iteration $n_{k}$ players ( $k$ and its subordinates) have left and that all these players paid compensation $A_{k}$, while $a_{p(k)}=d_{p(k)}+A_{k}$. Further note that $h$ is

\footnotetext{
${ }^{9}$ This property is called structural monotonicity in van den Brink and Gilles (1996) who use it to axiomatize the conjunctive (Shapley) permission value for permission restricted games.
} 
either $p(k)$ itself, or it is a superior of $p(k)$ and that $A_{h}=\frac{1}{n_{h}+1} \sum_{i \in F_{T}^{0}(h)} a_{i}$. From this and $A_{h} \geq A_{k}$ it follows that

$$
\tau_{h}(M, T(M), a)=\frac{\left(n_{h}+1\right) A_{h}-n_{k} A_{k}}{n_{h}-n_{k}+1} \geq \frac{\left(n_{h}+1\right) A_{h}-n_{k} A_{h}}{n_{h}-n_{k}+1}=A_{h} \geq A_{k} .
$$

So, at the second iteration we have for every $h \in M \backslash\{1\}$ that $\tau_{h}(M, T(M), a)$ is at least equal to the compensation $A_{k}$ assigned to $k$ and each of its subordinates in the first iteration. So, the players that leave in the second iteration have to pay at least the same amount as the players that have left in the first iteration.

Continuing in this way we obtain that the assigned payoffs are non-increasing in the iterations. The result now follows from the fact that for any two players $k$ and $h$ with $k$ a subordinate of $h$, player $h$ gets assigned its payoff either in the same iteration as $k$ or in a later iteration.

\section{Axioms}

In this section, we propose three axioms of a compensation scheme for liability problems with rooted-tree structure. These properties are inspired from the observations concerning tort law and case-system consistency, see Ito (1978). We then prove that the Nucleolus compensation scheme is the unique compensation scheme on the class $\mathcal{L}$ of liability problems with rooted-tree structure that satisfies the three properties. Without loss of generality, we assume that for every $(N, T, d) \in \mathcal{L}$ it holds that $1 \in N$ is the top of root $(N, T)$.

The first axiom takes into account the tort law principle that all tortfeasors are held jointly responsible for the total damage $d_{N}=\sum_{j \in N} d_{j}$, and therefore every tortfeasor should pay a fair share of this total damage. According to this principle the first property sets a uniform lower bound on the compensation to be paid by an individual tortfeasor. The axiom is obtained by considering for tortfeasor $i \in N \backslash\{1\}$ the additional damage $e_{i}=\sum_{k \in F_{T}^{0}(i)} d_{k}$ that has been caused by agent $i$ and his subordinates. Without the wrongful act of agent $i$ 's predecessor $p(i)$, the sequentially wrongful acts by agent $i$ and his subordinates would not have occurred. From this viewpoint, all agents in $F_{T}^{0}(i)$ and $p(i)$ are jointly responsible for the additional damage $e_{i}$ of agent $i$. We assume the case of torts where the causal weights between the agents in $F_{T}^{0}(i)$ and $p(i)$ cannot be determined and therefore a per capita criterion is justified. ${ }^{10}$ For $i \in N \backslash\{1\}$, this yields the per capita

\footnotetext{
${ }^{10}$ For instance, Ni and Wang (2007) use a per capita criterion to introduce the upstream equal responsibility sharing method for polluted river problems.
} 
contribution $\tau_{i}(N, T, d)$ given by

$$
\tau_{i}(N, T, d)=\frac{e_{i}}{\left|F_{T}^{0}(i)\right|+1},
$$

i.e., $\tau_{i}(N, T, d)$ is the equal division of the additional damage $e_{i}$ between $i$, all his subordinates and his predecessor.

Now, the first axiom requires that every tortfeasor $i$ should pay at least the smallest per capita contribution $\min _{j \in N \backslash\{1\}} \tau_{j}(N, T, d)$, i.e., the smallest per capita contribution is considered as a guarantee of every injuring party's compensation for the total damage.

Axiom 1 (Uniform lower bound) A compensation scheme $\varphi$ on $\mathcal{L}$ satisfies the uniform lower bound if for every $(N, T, d) \in \mathcal{L}$ and every $i \in N$,

$$
\varphi_{i}(N, T, d) \geq \min _{j \in N \backslash\{1\}} \tau_{j}(N, T, d)
$$

We stress that this is a very weak lower bound. Note that it does not require that $i$ contributes at least its own per capita contribution, but the minimal per capita contribution over all tortfeasors (except the first tortfeasor). In Example 3.6 it requires that every tortfeasor pays at least the per capita contribution of agent 5, being 14 .

The second axiom puts for every tortfeasor an individual upper bound on his compensation. Also this axiom is obtained by considering for tortfeasor $i \in N \backslash\{1\}$ the additional damage $e_{i}$ that has been caused by agent $i$ and his subordinates. Again this damage would not have occurred without the wrongful act of agent $i$ 's predecessor $p(i)$. From this viewpoint, agent $i$ and his predecessor can be held jointly responsible for the damage $e_{i}$. Assuming again the case of torts, the causal weights between $i$ and $p(i)$ cannot be determined. This yields an equal division of $e_{i}$ between $i$ and $p(i)$. The second axiom requires that every tortfeasor $i \neq 1$ should be held responsible for at most the half of his additional damage $e_{i}$. Since agent 1 has no predecessor, he should be held responsible for at most his full additional damage $e_{1}$, which is equal to the total damage $d_{N}$.

Axiom 2 (Individual upper bounds) A compensation scheme $\varphi$ on $\mathcal{L}$ satisfies the individual upper bounds if for every $(N, T, d) \in \mathcal{L}$ and every $i \in N \backslash\{1\}$,

$$
\varphi_{i}(N, T, d) \leq \frac{1}{2} e_{i}
$$

In Example 3.6 the vector of additional damages is given by $e=\left(e_{1}, e_{2}, e_{3}, e_{4}, e_{5}\right)=$ $(130,88,40,36,42,30)$, so the upper bounds for agents 2 to 6 are, respectively, 44, 20, 18, 21 and 15 . 
The third and last axiom is a type of case system consistency. Given a compensation scheme $\varphi$, consider a liability problem $(N, T, d) \in \mathcal{L}$ and a tortfeasor $i \in L(T)$ (thus a tortfeasor that does not have subordinates). Since such a tortfeasor can be held responsible for at most his own damage $d_{i}$, we may assume that he has to pay at most $d_{i}$. Now consider the related liability problem $\left(N \backslash\{i\}, T(N \backslash\{i\}), d^{i}\right)$, where $d^{i} \in \mathbb{R}^{N}$ is the vector of damages given by $d_{p(i)}^{i}=d_{p(i)}+d_{i}-\varphi_{i}(N, T, d)$ and $d_{k}^{i}=d_{k}$ for every $k \neq p(i)$ in $N \backslash\{i\}$. So, this is the liability problem obtained from $(N, T, d)$ by removing leaf $i$ and by adding the remaining damage $d_{i}-\varphi_{i}(N, d, T)$ not paid by $i$ to the damage $d_{p(i)}$ caused by his predecessor $p(i)$. The consistency axiom requires that the outcome chosen by a compensation scheme for every agent $j \in N \backslash\{i\}$ should be invariant under the departure of a leaf $i \in L(T)$ of the tree.

Axiom 3 (Leaf consistency) A compensation scheme $\varphi$ on $\mathcal{L}$ satisfies leaf consistency if for every $(N, T, d) \in \mathcal{L}$, every $i \in L(T)$ and every $j \in N \backslash\{i\}$

$$
\varphi_{j}\left(N \backslash\{i\}, T(N \backslash\{i\}), d^{i}\right)=\varphi_{j}(N, T, d) .
$$

In Example 3.6, leaf consistency, for example, implies that when agent 6 leaves and pays its nucleolus contribution 14, and we consider the new damage vector where the damage of agent 5 is $d_{5}+d_{6}-14=42-14=28$ and the other damages do not change, and in the structure we just leave agent 6 , then the nucleolus payoffs of the other agents do not change.

\section{Characterization of the Nucleolus compensation scheme}

In this section, we show that there is a unique compensation scheme on the class $\mathcal{L}$ of liability problems that satisfies the Axioms 1-3 and that this is the Nucleolus compensation scheme as defined in Definition 3.2. First, note that for an agent $i \in L(T), e_{i}=d_{i}$, and thus for $i \in L(T)$, Axiom 2 requires that $\varphi_{i}(N, T, d) \leq \frac{1}{2} d_{i}$. The next lemma states that when a compensation scheme satisfies Axiom 3 and the individual upper bound requirement for all leafs, then it also satisfies the individual upper bound requirement for all the other agents. In fact, the condition for the leafs and Axiom 3 imply Axiom 2 (for all agents).

Lemma 5.1 Let $\varphi$ on $\mathcal{L}$ be a compensation scheme that satisfies Axiom 3 and $\varphi_{i}(N, T, d) \leq$ $\frac{1}{2} d_{i}$ for every $i \in L(T)$. Then $\varphi$ satisfies Axiom 2 .

Proof. We prove that the upper bound holds for every other agent not being a leaf. Let $j$ be a player such that $j \in N \backslash(\{1\} \cup L(T))$. Let $d^{\prime} \in \mathbb{R}^{N}$ such that $d_{i}^{\prime}=d_{i}$ for $i \in N \backslash F_{j}^{0}(T)$ 
and $d_{j}^{\prime}=e_{j}-\sum_{k \in F_{T}(j)} \varphi_{k}(N, T, d) \leq e_{j}$. By subsequently applying Axiom 3 for all players in $F_{T}(j)$, it follows that $\left(N \backslash F_{T}(j), T\left(N \backslash F_{T}(j)\right), d^{\prime}\right) \in \mathcal{L}$ and

$$
\varphi_{j}(N, T, d)=\varphi_{j}\left(N \backslash F_{T}(j), T\left(N \backslash F_{T}(j)\right), d^{\prime}\right) .
$$

Since $j$ is a leaf on the subtree $\left(N \backslash F_{T}(j), T\left(N \backslash F_{T}(j)\right)\right.$, it follows that

$$
\varphi_{j}\left(N \backslash F_{T}(j), T\left(N \backslash F_{T}(j)\right), d^{\prime}\right) \leq \frac{1}{2} d_{j}^{\prime} \leq \frac{1}{2} e_{j}
$$

The next theorem states that the Nucleolus compensation scheme is the unique compensation scheme that satisfies Axiom 1 and 3 and the individual upper bounds (of Axiom 2) for the leafs. It is the main step to reach our main theorem.

Theorem 5.2 Let $\varphi$ on the class $\mathcal{L}$ of liability problems be a compensation scheme that satisfies Axioms 1 and 3 and for every $(N, T, d) \in \mathcal{L}$ the individual upper bound requirement $\varphi_{i}(N, T, d) \leq \frac{1}{2} e_{i}$ for every $i \in L(T)$. Then $\varphi(N, T, d)=N u c(N, T, d)$.

Proof. We prove that for every liability problem, the Axioms 1 and 3 and the upper bound requirements for the leafs determine the outcome computed by the Nucleolus algorithm. To simplify notation, let $x=\varphi(N, T, d)$. Without loss of generality, it is assumed that 1 is the root of the tree $(N, T)$. We subsequently consider liability problems with respectively $|N|=2,|N|=3$ and $|N|>3$.

Case 1, $|N|=2$. Consider a liability problem $(N, T, d) \in \mathcal{L}$ with $|N|=2$. Then, by Axiom 1 it follows that $x_{2} \geq \tau_{2}(N, T, d)=\frac{1}{2} d_{2}$ and by the upper bound requirement that $x_{2} \leq \frac{1}{2} e_{2}=\frac{1}{2} d_{2}$. Hence $x_{2}=\frac{1}{2} d_{2}$, and thus $x_{1}=d_{1}+\frac{1}{2} d_{2}$. This is equal to the outcome computed by the Nucleolus algorithm.

Case 2, $|N|=3$. For a liability problem $(N, T, d) \in \mathcal{L}$ with $|N|=3$ there are two possibilities: either $(N, T)$ is a line tree (one branch) or $(N, T)$ is a tree with two branches.

Case 2-1. First, we consider a line tree. Without loss of generality, we assume that $p(2)=1$ and $p(3)=2$. Now either $\frac{d_{3}}{2} \leq \frac{d_{2}+d_{3}}{3}$ or not.

Case 2-1-1, $\frac{d_{3}}{2} \leq \frac{d_{2}+d_{3}}{3}$. By Axiom 1 and the individual upper bound requirement for leaf 3 , it follows that $x_{3}=\frac{d_{3}}{2}$. Next, it follows by Axioms 1 and 3 and the uniform lower bound for leaf 2 in the subtree after removing agent 3 that $x_{2}=\frac{d_{2}+d_{3}-x_{3}}{2}$, and finally $x_{1}=d_{N}-x_{2}-x_{3}$. So $x$ is equal to the outcome computed by the Nucleolus algorithm.

Case 2-1-2, $\frac{d_{3}}{2}>\frac{d_{2}+d_{3}}{3}$. By Axiom 1, we must have that $x_{j} \geq \frac{d_{2}+d_{3}}{3}$ for $j=1,2,3$. The individual upper bound for leaf 3 requires that $x_{3}=\frac{d_{3}}{2}-c$ for some $c \geq 0$, and thus $\frac{d_{3}}{2}-c \geq \frac{d_{2}+d_{3}}{3}$. This yields $c \leq \frac{d_{3}}{6}-\frac{d_{2}}{3}$. By Axioms 1 and 3 and the uniform lower bound for leaf 2 in the subtree after removing agent 3 , it follows that $x_{2}=\frac{d_{2}+d_{3}-x_{3}}{2}$, and thus 
$x_{2}=\frac{d_{2}+d_{3}-x_{3}}{2} \geq \frac{d_{2}+d_{3}}{3}$. Substituting $x_{3}=\frac{d_{3}}{2}-c$ in this inequality gives $c \geq \frac{d_{3}}{6}-\frac{d_{2}}{3}$ and thus $c=\frac{d_{3}}{6}-\frac{d_{2}}{3}$. This implies that $x_{2}=x_{3}=\frac{d_{2}+d_{3}}{3}$ and thus $x_{1}=d_{1}+\frac{d_{2}+d_{3}}{3}$. Again $x$ is equal to the outcome computed by the Nucleolus algorithm.

Case 2-2. When $(N, T)$ is a tree with two branches we have $p(2)=p(3)=1$. Without loss of generality, let $d_{2} \geq d_{3}$. By Axiom 1 and the uniform lower bound for leaf 3 it follows that $x_{3}=\frac{d_{3}}{2}$. By Axiom 3 and case $|N|=2, x_{2}=\frac{d_{2}}{2}$ and thus $x_{1}=d_{1}+\frac{d_{2}}{2}+\frac{d_{3}}{2}$. Also in this case we have that $x$ is equal to the outcome computed by the Nucleolus algorithm.

Case 3, $|N|>3$. We now proceed with induction. Consider a liability problem $(N, T, d)$ with $|N|=n>3$. For any liability problem $\left(N^{\prime}, T^{\prime}, d^{\prime}\right) \in \mathcal{L}$ with $\left|N^{\prime}\right|<n$ we assume that $\varphi\left(N^{\prime}, T^{\prime}, d^{\prime}\right)=\operatorname{Nuc}\left(N^{\prime}, T^{\prime}, d^{\prime}\right)$. To show that then $x=N u c(N, T, d)$ we consider two possibilities, namely player $j$ with minimal $\tau_{j}(N, T, d)$ is either a leaf or not.

Case 3-1. There exists $i \in L(T)$ such that $\tau_{i}(N, T, d)=\frac{d_{i}}{2}=\min _{j \in N \backslash\{1\}} \tau_{j}(N, T, d)$. Take this leaf $i$ (if there are multiple, take an arbitrary one). By Axiom 1 and the individual upper bound for leaf $i$ it follows that $x_{i}=\frac{d_{i}}{2}$. This is also the outcome for $i$ as computed by the Nucleolus algorithm, and thus $x_{i}=N u c_{i}(N, T, d)$. Set $N^{\prime}=N \backslash\{i\}$ and take $d^{\prime} \in \mathbb{R}^{N^{\prime}}$ with $d_{j}^{\prime}=d_{j}$ for $j \in N^{\prime} \backslash\{p(i)\}$ and $d_{p(i)}^{\prime}=d_{p(i)}+d_{i}-x_{i}=d_{p(i)}+d_{i}-N u c_{i}(N, T, d)$.

Consider the lower bound liability game $\left(N, v_{L}\right)$ corresponding to $(N, L, d)$. Then the lower bound liability game corresponding to $\left(N^{\prime}, T\left(N^{\prime}\right), d^{\prime}\right)$ is the game $\left(N^{\prime}, v_{L}^{\prime}\right)$ where $v_{L}^{\prime}$ is given by setting for $S \subseteq N^{\prime}$,

$$
v_{L}^{\prime}(S)= \begin{cases}v_{L}(S \cup\{i\})-N u c_{i}(N, T, d) & \text { if } p(i) \in S, \\ v_{L}(S) & \text { otherwise. }\end{cases}
$$

It can be shown that $\left(N^{\prime}, v_{L}^{\prime}\right)$ is the Davis-Maschler reduced game of $\left(N, v_{L}\right)$ on $N^{\prime}$ with respect to $\operatorname{Nuc}(N, L, d) .{ }^{11} \quad$ By this observation and the fact that the nucleolus satisfies Davis-Maschler consistency ${ }^{12}$, it holds that for every $j \in N^{\prime}$ that $N u c_{j}(N, T, d)=$ $N u c_{j}\left(N^{\prime}, T\left(N^{\prime}\right), d^{\prime}\right)$. Further, for every $j \in N^{\prime}$, we have by the induction hypothesis

\footnotetext{
${ }^{11}$ For a game $(N, v) \in \mathcal{G}$, a vector $x \in \mathbb{R}^{N}$ and non-empty subset $N^{\prime}$ of $N$, the Davis-Maschler reduced game (Davis and Maschler, 1965) on $N^{\prime}$ with respect to $(N, v)$ and $x$ is the game $\left(N^{\prime}, w^{x}\right) \in \mathcal{G}$ defined by setting for all $S \subseteq N^{\prime}$,

$$
w^{x}(S)= \begin{cases}v(N)-\sum_{i \in N \backslash N^{\prime}} x_{i} & \text { if } S=N^{\prime}, \\ \max _{T \subseteq N \backslash N^{\prime}}\left[v(S \cup T)-\sum_{i \in T} x_{i}\right] & \text { if } S \neq N^{\prime}, \emptyset, \\ 0 & \text { if } S=\emptyset .\end{cases}
$$

${ }^{12}$ A solution $f$ on a subclass $\mathcal{G}^{\prime}$ of $\mathcal{G}$ satisfies Davis-Maschler consistency (Davis and Maschler, 1965) if for all $(N, v) \in \mathcal{G}^{\prime}$ and every non-empty $N^{\prime} \subset N$ it holds for $x=f(N, v)$ that the DM reduced game $\left(N^{\prime}, w^{x}\right) \in \mathcal{G}^{\prime}$ and $x_{N^{\prime}}=f\left(N^{\prime}, w^{x}\right)$, where $x_{N^{\prime}} \equiv\left(x_{i}\right)_{i \in N^{\prime}}$.
} 
that $\varphi_{j}\left(N^{\prime}, T\left(N^{\prime}\right), d^{\prime}\right)=N u c_{j}\left(N^{\prime}, T\left(N^{\prime}\right), d^{\prime}\right)$. Hence, for every $j \in N^{\prime}$ it holds that $N u c_{j}(N, T, d)=\varphi_{j}\left(N^{\prime}, T\left(N^{\prime}\right), d^{\prime}\right)$. With Axiom 3, it now follows that for every $j \in N^{\prime}$, $x_{j}=\varphi_{j}\left(N^{\prime}, T\left(N^{\prime}\right), d^{\prime}\right)=N u c_{j}(N, T, d)$.

Case 3-2: For all $i \in L(T)$, it holds that $\min _{j \in N \backslash\{1\}} \tau_{j}(N, T, d)<\tau_{i}(N, T, d)=\frac{d_{i}}{2}$. Let $k$ be such that $\tau_{k}(N, T, d)=\min _{j \in N \backslash\{1\}} \tau_{j}(N, T, d)$ and take some $i \in L(T)$ such that $i \in F_{T}^{0}(k)$, thus $i$ is subordinate of $k$. Since $i \neq k$, we have that also $p(i) \in F_{T}^{0}(k)$. From the Nucleolus algorithm we obtain that

$$
N u c_{i}(N, T, d)=N u c_{p(i)}(N, T, d)=\tau_{k}(N, T, d)
$$

By Axiom 1, we have that $x_{i} \geq \tau_{k}(N, T, d)=N u c_{i}(N, T, d)$. Suppose that $x_{i}>N u c_{i}(N, T, d)$. Set $N^{\prime}=N \backslash\{i\}$ and take $d^{\prime} \in \mathbb{R}^{N^{\prime}}$ with $d_{j}^{\prime}=d_{j}$ for $j \in N^{\prime} \backslash\{p(i)\}$ and $d_{p(i)}^{\prime}=d_{p(i)}+d_{i}-x_{i}$. By Axiom 3, we have that

$$
x_{p(i)}=\varphi_{p(i)}\left(N^{\prime}, T\left(N^{\prime}\right), d^{\prime}\right)
$$

and by the induction hypothesis,

$$
\varphi_{p(i)}\left(N^{\prime}, T\left(N^{\prime}\right), d^{\prime}\right)=N u c_{p(i)}\left(N^{\prime}, T\left(N^{\prime}\right), d^{\prime}\right) .
$$

Take $d^{\prime \prime} \in \mathbb{R}^{N^{\prime}}$ with $d_{j}^{\prime \prime}=d_{j}$ for $j \in N^{\prime} \backslash\{p(i)\}$ and $d_{p(i)}^{\prime \prime}=d_{p(i)}+d_{i}-N u c_{i}(N, T, d)$. Since by assumption $x_{i}>N u c_{i}(N, T, d)$, and thus $d_{p(i)}^{\prime}<d_{p(i)}^{\prime \prime}$, it follows from applying the Nucleolus algorithm that

$$
N u c_{p(i)}\left(N^{\prime}, T\left(N^{\prime}\right), d^{\prime}\right)<N u c_{p(i)}\left(N^{\prime}, T\left(N^{\prime}\right), d^{\prime \prime}\right)
$$

By the fact that the lower bound liability game corresponding to $\left(N^{\prime}, T\left(N^{\prime}\right), d^{\prime \prime}\right)$ is the Davis-Maschler reduced game of $\left(N, v_{L}\right)$ on $N^{\prime}$ with respect to $N u c(N, T, d)$, it follows with Davis-Maschler consistency that

$$
N u c_{p(i)}\left(N^{\prime}, T\left(N^{\prime}\right), d^{\prime \prime}\right)=N u c_{p(i)}(N, T, d)
$$

From the (in)equalities (5.2)-(5.6) it follows that

$$
x_{p(i)}<N u c_{p(i)}(N, T, d)=\tau_{k}(N, T, d)
$$

which contradicts Axiom 1. Therefore, for every $i \in L(T) \cap F_{T}^{0}(k), x_{i}=N u c_{i}(N, T, d)$. For every $i \in L(T) \cap F_{T}^{0}(k)$, let $N^{\prime}=N \backslash\{i\}$. Then it follows similar as above and from Axiom 3 that for every $j \in N^{\prime}, x_{j}=N u c_{j}(N, T, d)$.

Theorem 5.2 shows that if a compensation scheme satisfies Axioms 1 and 3 and the individual upper bound for the leafs, then it must be the nucleolus. On the other hand, we 
argue that the Nucleolus compensation scheme satisfies the Axioms 1, 2 and 3. First, from Lemma 3.7 and the algorithm it follows that the nucleolus compensation scheme satisfies Axiom 1. From the proof of Theorem 5.2 it follows that then Nucleolus compensation scheme satisfies Axiom 3 and the individual upper bound for the leafs. It then follows by Lemma 5.1 that Axiom 2 is satisfied. This yields our main result.

\section{Main result}

A compensation scheme $\varphi$ on the class $\mathcal{L}$ of liability problems satisfies Axioms 1, 2 and 3 if and only if $\varphi(N, T, d)=\operatorname{Nuc}(N, T, d)$.

The main result says that there is a unique compensation scheme supported by tort law bounds on the compensations (Axioms 1 and 2) and a type of case-system consistency (Axiom 3). This compensation scheme assigns to every liability problem the Rawlsian outcome given by the nucleolus. Finally, we check logical independence of the three axioms.

- Let $\varphi^{1}$ be the compensation scheme that assigns to every $(N, T, d) \in \mathcal{L}$ compensations given by $\varphi_{i}^{1}(N, T, d)=\min _{j \in N \backslash\{1\}} \tau_{j}(N, T, d)$ for every $i \neq 1$ and $\varphi_{1}^{1}(N, T, d)=$ $d_{N}-\sum_{i \neq 1} \varphi_{i}^{1}(N, T, d)$. Then $\varphi^{1}$ satisfies Axioms 1 and 2 , but not Axiom 3.

- Let $\varphi^{2}$ be the compensation scheme that assigns to every $(N, T, d) \in \mathcal{L}$ compensations given by $\varphi_{i}^{2}(N, T, d)=d_{i}$, for every $i \in N$. Then $\varphi^{2}$ satisfies Axioms 1 and 3 , but not Axiom 2.

- Let $\varphi^{3}$ be the compensation scheme given by $\varphi^{3}(N, T, d)=S h\left(N, v_{L}\right)$ for every $(N, T, d) \in \mathcal{L}$. Then $\varphi^{3}$ satisfies Axiom 2 and Axiom 3 (see Katsev, 2009), but not Axiom 1.

\section{Concluding remarks}

In this paper we considered the Nucleolus compensation scheme as a compensation scheme for liability problems, where causation of the cumulative injury results from multiple sequences of wrongful acts by different parties. It appears that the Nucleolus compensation scheme of a liability problem can be simply computed by using an algorithm for the nucleolus of a corresponding liability game. A very appealing property of the Nucleolus compensation scheme is that with respect to the additional damages of the coalitions, the smallest cost saving over all coalitions is made as large as possible, then the second smallest is made as large as possible, then the third smallest, and so on.

We also characterized the nucleolus compensation scheme by three axioms: a uniform lower bound, an individual upper bound and an axiom on case-system consistency. All these 
three axioms are derived from stylized facts concerning tort law. As noticed in Section 3, the class of lower-bound liability games is equivalent to the class of peer-group games. For this latter class Katsev (2009, chapter 6) provides a characterization of the Shapley value, namely the Shapley value is the unique efficient allocation rule on the class of peergroup games that satisfies leaf consistency, the weak veto property, top monotonicity and independence of non-subordinates. ${ }^{13}$ All these five axioms (including efficiency) are logically independent. The weak veto property states that player $i$ pays at least the same as any other player when $d_{j}=0$ for every $j \neq i$. Top monotonicity states that top player 1 pays at least the same amount as any other player. Finally independendence of non-subordinates states that if the damage of only one player $i$ changes, then the compensations to be paid by the subordinates of $i$ do not change.

We are now ready to compare the Shapley compensation scheme and the nucleolus compensation scheme by their sets of characterizing axioms. It is easy to verify that also the Nucleolus compensation scheme satisfies the weak veto property and top monotonicity, but not independence of non-subordinates. So, the Nucleolus compensation scheme satisfies all above Shapley axioms, except the independence of non-subordinates. On the other hand the Shapley compensation scheme satisfies leaf consistency and the individual upper bound, but not the uniform lower bound. So, the two solutions have in common that both satisfy leaf consistency, individual upper bound, the weak veto property and top monotonicity. Then the Nucleolus compensation scheme satisfies uniform lower bound but not independence of non-subordinates, whereas the Shapley compensation scheme satisfies the latter but not the former. Although the two solutions differ in only one axiom, the differences in the actual outcome for a specific liability problem might be quite big. When $i$ is a leaf of the tree, then equation (3.1) shows that according to the Shapley compensation scheme the damage $d_{i}$ is equally shared by $i$ and all its superiors. In particular, in a linear tree with $n$ tortfeasors the last one pays only a share $\frac{1}{n}$ of its own damage $d_{i}$. On the other hand, according to the Nucleolus compensation scheme the share of a leaf $i$ in its own damage $d_{i}$ can be $\frac{1}{2}$ and this indeed happens when $d_{i}$ is relatively small compared to the damages of its superiors. In fact, for a linear tree with $n$ tortfeasors the uniform lower bound implies that the damage $d_{n}$ of leaf $n$ is shared equally amongst all tortfeasors when $d_{j}=0$ for every superior $j$ of leaf $n$, whereas together with the individual upper bound it also implies that $n$ pays half of its own damage when $d_{n}$ is small compared to the other damages. So, while according to the Shapley compensation leaf $n$ is always held responsible for precisely share $\frac{1}{n}$ of its own damage, according to the Nucleolus compensation scheme

\footnotetext{
${ }^{13}$ Also in Ferey and Dehez (2015) an axiomatization of the Shapley value on the class of (linear) lowerbound liability games is given. In fact, in this axiomatization leaf consistency, the weak veto property and top monotonicity are replaced by the single axiom of zero immediate damage, saying that a player $i$ pays the same as its predecessor $p(i)$ if the damage of the predecessor is equal to zero.
} 
the responsibility of a leaf for its own damage is $\frac{1}{2}$ when his damage is small, but it might decrease to a share of only $\frac{1}{n}$ (as in the Shapley compensation scheme) when his damage is large compared to the damages of his superiors. In fact, we have seen in Section 3 that both the Shapley compensation scheme and the nucleolus compensation scheme satisfy weak monotonicity, saying that a tortfeasor never pays a higher compensation than his predecessor.

Finally, we discuss what happens when the population of the tortfeasors is increasing. Suppose that a new tortfeasor arrives at the end of a branch of the tree, so he is added as new leaf to one of the leafs of the existing tree. One may wonder whether every original tortfeasor pays in the new situation at least the same as in the original situation. If the answer for this question is negative, then it might be that some of the original tortfeasors have an incentive to increase the population of the tortfeasors, which leads to an increase of the total damage. From this aspect, it is appropriate to require that a compensation scheme satisfies leaf population monotonicity, stating that when a new tortfeasor arrives, in the new situation every original tortfeasor pays at least the same as in the original situation. If this property is satisfied, then no original tortfeasor has an incentive to increase the population of the tortfeasors.

From equation (3.1) it follows immediately that the Shapley value satisfies the leaf population property. When a new tortfeasor arrives, then his (additional) damage is equally shared amongst himself and his superiors with no effect on how the other damages are shared. So, the question is whether also the Nucleolus compensation scheme satisfies leaf population monotonicity. This answer is affirmative and follows from a result in Katsev (2009, chapter 6), who shows that when $d$ and $d^{\prime}$ are such that $d_{i}^{\prime} \geq d_{i}$ for every $i \in N$, then every tortfeasor should pay at $d^{\prime}$ at least the same as at $d$. So, for a given tree with a fixed set of tortfeasors, the compensations to be paid by the tortfeasors are non-decreasing in the damages. ${ }^{14}$ Now, suppose a new tortfeasor is added. Then, according to the Nucleolus compensation scheme this tortfeasor pays at most half of its damage and leaf consistency says that the others have to pay according to the original situation, but with the damage of the predecessor of the new leaf replaced by the sum of its own damage and the remaining part of the damage of the new tortfeasor. So, the new situation reduces to the old situation, but with higher damage for the predecessor of the new leaf. So, by the result of Katsev it follows that every original tortfeasor pays at least the same as before. Hence also the Nucleolus compensation scheme satisfies leaf population monotonicity.

\footnotetext{
${ }^{14}$ In the literature this is known as resource monotonicity, but here the resources are the damages.
} 


\section{References}

[1] Boston, G. W. "Apportionment of harm in tort law: a proposed restatement." University of Dayton Law Review 21, 267-378. 1995-1996.

[2] Brânzei, R, V. Fragnelli, S. Tijs. "Three connected line graph peer group situations and line graph peer group games." Mathematical Methods of Operations Research, 55, 93-106, 2002.

[3] Brânzei, R, T. Solymosi, S. Tijs. "Strongly essential coalitions and the nucleolus of peer group games." International Journal of Game Theory, 33, 447-460, 2005.

[4] Brink, R, van den. "An axiomatization of the Shapley value using a fairness property." International Journal of Game Theory 30, 309-319, 2001.

[5] Brink, R, van den, and R.P. Gilles. "Axiomatizations of the conjunctive permission value for games with permission structures." Games and Economic Behavior 12, 113126.

[6] Brink, R, van den, P.J.J. Herings, G. van der Laan, A.J.J. Talman. "The average tree permission value for games with permission structure." Economic Theory 58, 99-123, 2015.

[7] Brink, R, van den, C. Dietz, G. van der Laan, G. Xu. "Comparable characterizations of four solutions for permission tree games." Tinbergen Institute Discussion Paper TI 2015-021/II, 2015. (forthcoming in Economic Theory).

[8] Davis, M, M. Maschler. "The kernel of a Cooperative Game." Naval Research Logistics Quarterly 12, 223-259, 1965.

[9] Dehez, P, S. Ferey. "How to share joint liability: a cooperative game approach." Mathematical Social Sciences 66, 44-50, 2013.

[10] S. Ferey, P. Dehez. "Multiple causation, apportionment and the Shapley value." Core Discussion Paper 2015/16, 2015.

[11] Gilles, R. P, G. Owen, R. van den Brink. "Games with permission structures: the conjunctive approach." International Journal of Game Theory 20, 277-293, 1992.

[12] Ito, M. "An introductory remark." Jurist 59, 6-7, 1978. (in Japanese)

[13] Kalai, E, D. Samet. "On weighted Shapley values." International Journal of Game Theory 16, 205-222, 1987. 
[14] Katsev, I. Solutions for Games with Restricted Cooperation. Ph D. thesis, Free University, Amsterdam, 2009.

[15] Landes, W.M, R.A. Posner. "Joint and multiple tortfeasors: an economic analysis". Journal of Legal Studies 9, 517-555, 1980.

[16] Littlechild S.C, G. Owen. "A simple expression for the Shapley value in a special case". Management Science 3, 370-372, 1973.

[17] Moulin H.V. Fair Division and Collective Welfare. MIT Press, 2003.

[18] Myerson, R.B. "Conference structure and fair allocation rules." International Journal of Game Theory 9, 169-182, 1980.

[19] Ni, D, Y. Wang. "Sharing a polluted river". Games and Economic Behavior 60: 176$186,2007$.

[20] Oishi, T, M. Nakayama. "Anti-dual of economic coalitional TU games". Japanese Economic Review 60, 560-566, 2009.

[21] Oishi, T, M. Nakayama, T. Hokari, Y. Funaki. "Duality and anti-duality in TU games applied to solutions, axioms, and axiomatizations". Journal of Mathematical Economics 63, 44-53, 2016.

[22] Parisi, F, R. Singh. "The efficiency of comparative causation". Review of Law and Economics 6, 219-245, 2010.

[23] Rawls, J. A Theory of Justice, Oxford University Press, New York and Oxford, 1971.

[24] Schmeidler, D. "The nucleolus of a characteristic function game." SIAM Journal on Applied Mathematics 17, 1163-1170, 1969.

[25] Shapley L.S. "A value for $n$-person games." In: Kuhn H, Tucker A.W. (eds) Contributions to the theory of games II, Princeton University Press, pp.307-317, 1953.

[26] Shavell, S. "Torts in which victim and injurer act sequentially". Journal of Law and Economics 26, 589-612, 1983. 
Tinbergen Institute is the graduate school and research institute in economics of Erasmus University Rotterdam, the University of Amsterdam and VU University Amsterdam.

More TI discussion papers can be downloaded at http://www.tinbergen.nl

Tinbergen Institute has two locations:

Tinbergen Institute Amsterdam

Gustav Mahlerplein 117

1082 MS Amsterdam

The Netherlands

Tel.: +31(0)20525 1600

Tinbergen Institute Rotterdam

Burg. Oudlaan 50

3062 PA Rotterdam

The Netherlands

Tel.: +31(0)10 4088900

Fax: +31(0)10 4089031 


\title{
An axiomatic analysis of joint liability problems with rooted-tree structure ${ }^{1}$
}

\author{
Takayuki Oishi ${ }^{2} \quad$ Gerard van der Laan ${ }^{3} \quad$ René van den Brink ${ }^{4}$
}

May 25, 2016

\footnotetext{
${ }^{1}$ The authors would like to thank Shin Sakaue for his helpful comments.

${ }^{2}$ Department of Economics, Aomori Public University, e-mail: takayuki1q80@gmail.com

${ }^{3}$ Department of Econometrics and Operations Research, and Tinbergen Institute, VU University Amsterdam, e-mail: g.vander.laan@vu.nl

${ }^{4}$ Department of Econometrics and Operations Research, and Tinbergen Institute, VU University Amsterdam, e-mail: j.r.vanden.brink@vu.nl
} 


\begin{abstract}
For joint liability problems concerning tort law, a legal compensation scheme may be based on lower and upper bounds of compensation for injury and on case-system consistency. Introducing several properties inspired from this observation, we analyze compensation schemes axiomatically under the situation where causation of the cumulative injury appears in multiple sequences of wrongful acts. The situation underlying the model is described by a rooted-tree graph. We show that there is a unique compensation scheme that satisfies three axioms, one about lower bounds of individual compensations, one about upper bounds of individual compensations, and one about case-system consistency. This unique compensation scheme is the nucleolus of an associated liability game.
\end{abstract}

Keywords: Liability problems, Tort law, Rooted-tree graph, Axiomatization, Nucleolus, JEL Classification Number: D63, K13, K49 


\section{Introduction}

In this paper we consider situations in which an injured party suffers damages caused by wrongful acts performed subsequently by a sequence of injuring parties. The wrongful acts are causally related in the sense that any wrongful act in the sequence would not have occurred if any of the preceding wrongful acts would not have occurred. So, the second (wrongful) act can only occur after the first (wrongful) act has occurred, the third (wrongful) act can only occur when both the first and the second (wrongful) acts have occurred and so on. Any wrongful act results in an amount of damage to the injured party. The injuring parties are the tortfeasors who can be considered to be jointly liable for the full damage. The problem is how to apportion the full damage amongst the tortfeasors. In many real life situations, this problem is brought to court. This sharing problem is referred to as the Liability problem.

Historically, common law did not accept any apportionment among the tortfeasors, but evolution of common law in the 19th and 20th centuries led to the third Restatement of Torts (May 1999), providing basic principles and rules to apportion the damages. However, a systematic apportionment method is still the subject of research. In the existing literature on law and economics, it is a central topic to clarify whether or not a legal compensation scheme for liability problems is useful, see for instance Landes and Posner (1980), Shavell (1983), and Parisi and Singh (2010). These authors analyze the functioning of compensation schemes from the viewpoint of incentives. On the other hand, the viewpoint of fairness is also important. In fact, tort law prescribes an award of damages to achieve fair compensation for injury, see Boston (1995-1996). Although a few researchers have investigated the normative topic of liability problems (for instance, see Dehez and Ferey (2013) and Ferey and Dehez (2015)), this topic still needs further investigation.

In this paper, we analyze the functioning of compensation schemes from the viewpoint of fairness. For this purpose, we take an axiomatic approach. ${ }^{1}$ The axioms proposed in this paper are derived by taking into account tort law. Furthermore, we are interested in the situation where the determination of causal weights between the injuring parties is difficult in the following two senses. First, since a judge determines causal weights subjectively, fair compensation for injury may be difficult. Second, if the judge's transaction cost of this determination is high, then the determination may be impossible. Under this situation, we axiomatize the compensation scheme associated with the 'difference principle of social justice' à la Rawls (1971).

The liability problem as described in Dehez and Ferey (2013) has a linear structure in the sense that the agents are linearly ordered: the wrongful act of agent $i$ can only occur

\footnotetext{
${ }^{1}$ Axiomatic approaches to economic allocation problems, where the axioms are based on principles of distributive justice have been described in, e.g. Moulin (2003)
} 
when all agents $j<i$ behave wrongfully. In this paper, we consider the more general class of liability problems with rooted-tree structure. As an example, we consider the case where the injured party suffers an injury that is caused by four agents, agents 1, 2, 3, and 4 . Agent 1 has taken a wrongful act that is the root of the injury. After agent 1's wrongful act, agents 2 and 3 have taken wrongful acts. Without agent 1's wrongful act, agents 2 and 3's wrongful acts would not have occurred. On the other hand, agent 2's wrongful act does not affect agent 3's wrongful act and reversely. After agent's 1 wrongful act, the wrongful act of agent 3 might occur without the wrongful act of agent 2 and reversely, the wrongful act of agent 2 might occur without the wrongful act of agent 3 . Without the wrongful acts of both agents 1 and 2, agent 4's wrongful act would not have occurred. The situation underlying this example can be modeled by the rooted-tree graph of Figure 1 . In this graph, agent 1 is located at the root and has two branches, at one branch agent 1 is succeeded by agent 2 and agent 2 is succeeded by agent 4 , at the other branch agent 1 is succeeded by agent 3 . Agents 3 and 4 are the leafs of the tree. The rooted-tree represents the hierarchical structure of causation of the cumulative injury.

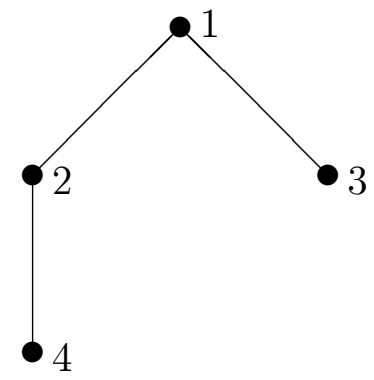

Figure 1: Rooted tree with two branches.

Formally, the liability problem is how to share the total damage amongst the $n$ tortfeasors, called the agents and indexed by $i=1, \ldots, n$. A compensation scheme is a sharing rule that determines for every liability problem the compensations that have to be paid by the individual tortfeasors. Note that a compensation scheme is a procedure (algorithm) that can be applied to every liability problem.

Following the existing literature, we use several notions of damages. Here, we explain the notions by using the case of liability problems with linear structure. Analogously to this case, it is easy to understand the notions of damages in the case of liability problems with rooted-tree structure. It is supposed that all damages can be measured monetary. Every agent $i$ has taken a wrongful act that causes an amount of damage $d_{i}$. However, the wrongful act of agent $i$ can only occur when all agents $1, \ldots, i-1$ have taken wrongful acts. So, if at least one of the wrongful acts of the agents $1, \ldots, i-1$ would not have occurred, 
then the wrongful act of agent $i$ would not have occurred. In the existing literature, see for instance Dehez and Ferey $(2013)^{2}$, the amount $d_{i}$ is called the direct damage resulting from the wrongful act of $i$. Furthermore, for every $i$ the cumulative damage up to $i$ is defined as $c_{i}=\sum_{k=1}^{i} d_{k}$ and the additional damage of $i$ is defined as $e_{i}=c_{n}-c_{i-1}=\sum_{k=i}^{n} d_{k}$. So, the cumulative damage up to agent $n$ is the total damage of the tortfeasors, while the additional damage of $i$ is the sum of all damages that would not have occurred without the wrongful act of $i$. Finally, we have the notion of potential damage. This type of damage is defined for every subset $S \subseteq\{1, \ldots, n\}$ of tortfeasors. Define $k(S)=0$ if agent 1 is not a member of $S$. Otherwise, define $k(S)$ as the highest indexed member of $S$ such that all consecutive agents $1, \ldots, k(S)$ also belong to $S$. Then the potential damage of $S$ is the cumulative damage $c_{k(S)}$, with $c_{0}=0$, i.e. it is sum of the damages that the members of $S$ cause when the members outside $S$ do not behave wrongfully.

Dehez and Ferey (2013) introduce a certain compensation scheme formalized by causal weights between the injuring parties and the list of additional damages. They show that for every liability problem this compensation scheme yields the (weighted) Shapley value of the corresponding transferable utility game (for short TU game) that assigns worth $v(S)=c_{k(S)}$ to every subset $S$ of tortfeasors, i.e., the worth of $S$ is the potential damage of $S$. The (weighted) Shapley value (Shapley 1953; Kalai and Samet 1987) is an established solution for TU games, and it is a game theoretic expression of fairness. Several notions of fairness underlying the Shapley value are proposed in the existing literature, for instance see Myerson (1980) and van den Brink (2001). In Ferey and Dehez (2015) a characterization of the compensation scheme yielding the Shapley value for liability problems is given.

As Dehez and Ferey (2013) point out, for a legal compensation scheme concerning tort law it should be required that for every liability problem it yields individual compensations that satisfy the following properties:

(i) Every injuring party should pay at least the potential damage that he would have caused alone.

(ii) Every injuring party should pay at most the additional damage that he would have caused. This principle is supported by the third Restatement of Torts, which is formulated by the American Law Institute.

For a liability problem with rooted-tree structure, based on these two properties we define in this paper two TU games, the lower-bound liability game and the upper-bound liability game. The first one assigns to every subset $S$ of tortfeasors a worth $v_{L}(S)$ that is equal to the potential damage of $S$. For problems with linear structure this game reduces to the liability game as defined in Dehez and Ferey (2013). The upper-bound liability game

\footnotetext{
${ }^{2}$ Here and in the sequel when we refer to Dehez and Ferey (2013), see also Ferey and Dehez (2015)
} 
assigns to every subset $S$ of tortfeasors a worth $v_{U}(S)$ that is equal to the additional damage of $S .^{3}$ It appears that these two games are the duals of each other in the game-theoretic sense.

A vector $x=\left(x_{1}, \ldots, x_{n}\right)$ of individual compensations, in the sequel shortly outcome, is in the core of the lower-bound game if for every $S$ it holds that $\sum_{i \in S} x_{i} \geq v_{L}(S)$, i.e., the total compensation to be paid by the members of $S$ is at least equal to their potential damage. Also, for every $S \subseteq N, x$ satisfies $\sum_{i \in S} x_{i} \geq v_{L}(S)$ if and only if $\sum_{i \in S} x_{i} \leq v_{U}(S)$, so an outcome in the core of the lower-bound liability game satisfies the Properties (i) and (ii) mentioned above. In fact, an outcome is in the core if and only if it satisfies this type of properties for every subset of tortfeasors. Generalizing a result of Dehez and Ferey (2013) for linear liability games, also for liability games with rooted tree structure the lower-bound liability game is convex.

In this paper, we propose three axioms and we show that these three axioms determine a unique compensation scheme that yields for every liability problem with rooted-tree structure an outcome in the core of the lower-bound liability game. As Property (i), the first axiom sets for every tortfeasor a uniform lower bound. This lower bound is the same for every tortfeasor, and for an individual tortfeasor the best possible outcome is an outcome in which he has to pay this lower bound. As Property (ii), the second axiom sets for every tortfeasor an individual upper bound. This upper bound differs over the tortfeasors and gives for every tortfeasor its worst possible outcome. In order to set the lower and upper bound axioms, per capita criteria are employed. This is because in liability problems it is often impossible to determine the causal weights between the injuring parties. In this situation, per capita criteria might be justified. The third and last axiom stems from the stylized fact that in the UK and America, a legal compensation scheme is based on so-called case system consistency, see for instance Ito (1978). This requires that the compensation scheme has the property that for every liability problem it provides an outcome that is consistent with the outcome that the same procedure generates for a different, but similar liability problem. In this paper, we propose leaf consistency. This type of case system consistency requires that for every liability problem with rooted-tree structure the compensation scheme is invariant when a leaf of the tree pays his compensation and leaves.

We show that the three axioms uniquely determine a compensation scheme. This scheme assigns to every liability problem with rooted-tree structure the so-called nucleolus of the lower bound liability game as outcome. The Nucleolus compensation scheme has two appealing properties.

First, the nucleolus (Schmeidler, 1969) is an established outcome for TU games. In fact, it is a game-theoretic expression of the 'difference principle of social justice' à la Rawls

\footnotetext{
${ }^{3}$ The additional damage of a subset $S$ of tortfeasors will be defined formally in the next section.
} 
(1971). So, when it is desirable that a legal compensation scheme for liability problems is to attain a Rawlsian outcome, the three axioms yield a useful compensation scheme.

Second, it is well known that the nucleolus of a game is in the core if the core is nonempty. Using the fact that the upper bound liability game is the dual of the lower bound liability game, it follows that the nucleolus of the lower bound liability game lexicographically maximizes the differences $v_{U}(S)-\sum_{i \in S} x_{i}$ of all subsets $S$ of tortfeasors, i.e. it makes the smallest difference over all subsets $S$ as high as possible, then the second smallest one, then the third smallest one, and so on. So, it maximizes (lexicographically) over all different subsets $S$ of tortfeasors the differences between the additional damage $v_{U}(S)$ and the actual compensation $\sum_{i \in S} x_{i}$ to be paid by the members of $S$. Loosely speaking, it minimizes (lexicographically) the dissatisfactions over the subsets of tortfeasors with respect to their 'worst-case' outcomes, i.e. the outcome in which $S$ has to pay the total amount $v_{U}(S)$ of its additional damage.

The remainder of the paper is organized as follows. In Section 2, preliminaries are given. In Section 3, the liability problem with rooted-tree structure and the corresponding liability games are given. In Section 4, we state and discuss the three axioms to be satisfied by a compensation scheme. In Section 5, we show that the compensation scheme that satisfies the axioms of Section 4 assigns to every liability problem the nucleolus of the corresponding lower-bound liability game. Section 6 contains concluding remarks, and discusses a comparison between the Shapley and nucleolus compensation schemes, and an incentive problem in the situation where the population of the tortfeasors is increasing.

\section{Preliminaries}

A cooperative game with transferable utility, or simply a TU game, is a pair $(N, v)$, where $N \subseteq \mathbb{N}$ is a finite set of players, and $v: 2^{N} \rightarrow \mathbb{R}$ is a characteristic function that assigns a worth $v(S) \in \mathbb{R}$ to every subset (usually called coalition) $S$ of $N$, satisfying $v(\emptyset)=0$. A TU game $(N, v)$ is convex if $v(S \cup T)+v(S \cap T) \geq v(S)+v(T)$ for all $S, T \subseteq N$. It is concave if these inequalities are reversed. We denote by $\mathcal{G}$ the class of all TU games. The subclass of all convex TU games is denoted by $\mathcal{G}^{\text {vex }}$ and the subclass of all concave TU games by $\mathcal{G}^{\text {cave }}$. For a game $(N, v) \in \mathcal{G}$, the dual game, denoted by $\left(N, v^{d}\right)$, assigns to every coalition $S$ what the 'grand coalition' $N$ loses if the players in $S$ stop cooperating, and thus is defined by $v^{d}(S)=v(N)-v(N \backslash S)$ for all $S \subseteq N$. Note that $v^{d}(\emptyset)=0$ and $v^{d}(N)=v(N)$. Further it holds that $(N, v) \in \mathcal{G}^{v e x}$ if and only if $\left(N, v^{d}\right) \in \mathcal{G}^{\text {cave }}$.

A payoff vector of TU game $(N, v)$ is a vector $x \in \mathbb{R}^{N}$ giving a payoff $x_{i} \in \mathbb{R}$ to every player $i \in N$. A payoff vector is efficient if $\sum_{i \in N} x_{i}=v(N)$. Given $(N, v) \in \mathcal{G}$, the 
preimputation set of $(N, v)$, denoted by $P I(N, v)$, is the set of all efficient payoff vectors. Further, the imputation set, denoted by $I(N, v)$, is the subset of all vectors in $P I(N, v)$ that satisfy $x_{i} \geq v(\{i\})$ for every $i \in N$ (individual rationality); the anti-imputation set, denoted by $A I(N, v)$, is the subset of all vectors in $P I(N, v)$ that satisfy $x_{i} \leq v(\{i\})$ for every $i \in N$. Note that these sets are not-empty if and only if $v(N) \geq \sum_{i \in N} v(\{i\})$, respectively $v(N) \leq \sum_{i \in N} v(\{i\})$. We denote by $\mathcal{G}^{I}$ the class of all TU-games with nonempty $I(N, v)$, and by $\mathcal{G}^{A I}$ the class of all TU-games with nonempty $A I(N, v)$. Note that $\mathcal{G}^{\text {vex }}$ is a subset of $\mathcal{G}^{I}$ and $\mathcal{G}^{\text {cave }}$ is a subset of $\mathcal{G}^{A I}$.

The core of a game $(N, v)$, denoted by $C(N, v)$, is the set of (efficient) payoff vectors that are group stable, and is given by

$$
C(N, v)=\left\{x \in P I(N, v) \mid \sum_{i \in S} x_{i} \geq v(S) \text { for all } S \subseteq N\right\} .
$$

Note that $C(N, v)$ is a subset of $I(N, v)$ and that it might be empty. Every game $(N, v) \in$ $\mathcal{G}^{\text {vex }}$ has a non-empty core. A vector $x \in C(N, v)$ satisfies the requirement that for every coalition $S$ the total payoff is at least equal to its own worth. This is reasonable when $(N, v)$ is a profit game, i.e., the worth $v(S)$ is the total revenue that the members of $S$ can achieve by cooperating. However, when $v$ is a cost game, i.e., coalition $S$ has costs $v(S)$ when it stands alone, then the worth should be considered as upper bounds on the contributions. For a cost game it makes sense to apply the anti-core of a game $(N, v)$, denoted by $A C(N, v)$. This set of efficient payoff vectors is given by

$$
A C(N, v)=\left\{x \in P I(N, v) \mid \sum_{i \in S} x_{i} \leq v(S) \text { for all } S \subseteq N\right\} .
$$

The anti-core $A C(N, v)$ is a subset of $A I(N, v)$ and might be empty, but every game $(N, v) \in \mathcal{G}^{\text {cave }}$ has a non-empty anti-core.

For a given subset $\mathcal{G}^{\prime}$ of the class $\mathcal{G}$ of all TU-games, a (single-valued) solution is a function $f$ that assigns to every game $(N, v)$ in $\mathcal{G}^{\prime}$ a payoff vector $f(N, v) \in P I(N, v)$. Note that in this paper we require that a solution assigns to each game an efficient payoff vector. The best-known solution on the class $\mathcal{G}$ of all TU-games is the Shapley value (Shapley, 1953), denoted $S h$. This solution assigns to every game $(N, v) \in \mathcal{G}$ the payoff vector $\operatorname{Sh}(N, v)$ given by ${ }^{4}$

$$
S h_{i}(N, v)=\sum_{S \subseteq N: i \in S} \frac{(|N|-|S|) !(|S|-1) !}{|N| !}(v(S)-v(S \backslash\{i\})) \text { for all } i \in N .
$$

So, for every player $i \in N$ the payoff is a weighted sum of its marginal contributions $v(S)-v(S \backslash\{i\})$ to the coalitions $S$ containing $i$. When $(N, v)$ is convex, then $S h(N, v) \in$

\footnotetext{
${ }^{4}$ For a finite set $A$, we denote by $|A|$ the number of elements in $A$ (cardinality of $A$ ).
} 
$C(N, v)$. However, in general, on the domain of TU games with non-empty cores it might be that the Shapley value is not in the core. Further it holds that the Shapley value is self-dual (see Kalai and Samet (1987)), saying that for every $(N, v) \in \mathcal{G}$ it holds that $\operatorname{Sh}\left(N, v^{d}\right)=\operatorname{Sh}(N, v) .^{5}$

Another well-known solution on the class of all TU-games is the prenucleolus. Given a TU game $(N, v) \in \mathcal{G}$, we define for payoff vector $x \in P I(N, v)$ and coalition $S \subseteq N$ the excess of $S$ with respect to $x$ as

$$
e(S, x, v) \equiv v(S)-\sum_{i \in S} x_{i}
$$

When the payoffs are revenues, i.e., the payoffs are payments to the players, the excess $e(S, x, v)$ can be seen as a measure of dissatisfaction of coalition $S$. The bigger the excess of $S$ and thus the bigger the difference between its own worth $v(S)$ and its payoff $\sum_{i \in S} x_{i}$ received by the members of $S$, the more dissatisfied coalition $S$ is. Now, let $\theta(x, v) \in \mathbb{R}^{2^{N}}$ be the vector obtained by arranging all the excesses in non-increasing order, so the first component of $\theta(x, v)$ is the excess of a coalition with the highest excess, the second component is the excess of a coalition with the highest excess under the remaining coalitions, and so on. Then the prenucleolus is the solution on $\mathcal{G}$ that assigns to every game $(N, v) \in \mathcal{G}$ the unique vector in $P I(N, v)$ that minimizes lexicographically the dissatisfactions. To be precise, the prenucleolus assigns to every game $(N, v)$ the unique payoff vector $x \in P I(N, v)$ such that for every $y \in P I(N, v) \backslash\{x\}$ there exist a component $k$ such that $\theta_{h}(x, v)=\theta_{h}(y, v)$ for all $h<k$, and $\theta_{k}(x, v)<\theta_{k}(y, v)$, i.e. the prenucleolus assigns to $(N, v) \in \mathcal{G}$ the unique payoff vector $x \in P I(N, v)$, such that for every other vector $y \in P I(N, v)$ there exists a number $k$ such that the $k-1$ biggest excesses of $x$ and $y$ are equal and the next biggest excess of $x$ is smaller than the next biggest excess of $y$. We denote the prenucleolus of a game $(N, v)$ by $P N u c(N, v)$.

The nucleolus is a solution defined for every game with $I(N, v)$ not-empty, so on the subclass of games $\mathcal{G}^{I}$. It assigns to every $(N, v) \in \mathcal{G}^{I}$ the unique individually rational payoff vector $x \in I(N, v)$ that minimizes lexicographically the dissatisfactions over all vectors in $I(N, v)$. We denote the nucleolus of a game $(N, v)$ by $\operatorname{Nuc}(N, v)$. When the core is nonempty, $\operatorname{Nuc}(N, v) \in C(N, v)$. Further, on the class of games with non-empty $C(N, v)$ the nucleolus coincides with the prenucleolus (Schmeidler, 1969). In particular it holds that $\operatorname{Nuc}(N, v)=P \operatorname{Nuc}(N, v)$ when $(N, v)$ is convex.

Similarly, for a game $(N, v)$ we define the anti-prenucleolus, denoted $A P N u c(N, v)$, and for a game $(N, v)$ in the subclass $\mathcal{G}^{A I}$ the anti-nucleolus, denoted $A N u c(N, v)$. The antiprenucleolus is the unique payoff vector $x \in P I(N, v)$ such that $-\theta(v, x)$ is lexicographically

\footnotetext{
${ }^{5}$ The notion of (self-)duality plays an important role in axiomatizing solutions for TU games. For instance, see Oishi, Nakayama, Hokari, and Funaki (2016).
} 
smaller than $-\theta(v, y)$ for every $y \in P I(N, v)$, i.e., it is the unique vector in $P I(N, v)$ that minimizes lexicographically the vector of negative excesses $\sum_{i \in S} x_{i}-v(S)$ for all $S \subseteq N$. So, for a cost game, the anti-prenucleolus lexicographically maximizes the cost savings $v(S)-\sum_{i \in S} x_{i}$ for all $S \subseteq N$. The anti-nucleolus assigns to every $(N, v) \in \mathcal{G}^{A I}$ the unique payoff vector $x \in I(N, v)$ that minimizes lexicographically the vector of negative excesses over all vectors in $A I(N, v)$. When the anti-core is non-empty, $\operatorname{ANuc}(N, v) \in A C(N, v)$. When $(N, v) \in \mathcal{G}^{\text {cave }}$ it holds that $\operatorname{ANuc}(N, v)=A P N u c(N, v)$.

In the next sections the following proposition, which follows from Oishi and Nakayama (2009), will appear to be useful. Recall that $(N, v) \in \mathcal{G}^{v e x}$ if and only if $\left(N, v^{d}\right) \in \mathcal{G}^{\text {cave }}$.

Proposition 2.1 For every $(N, v) \in \mathcal{G}^{v e x}$ it holds that

(i) $A C\left(N, v^{d}\right)=C(N, v)$,

(ii) $\operatorname{ANuc}\left(N, v^{d}\right)=\operatorname{Nuc}(N, v)$.

We now introduce rooted trees. First, a directed graph or digraph is a pair $(N, D)$, where $N$ is a set of nodes and the collection of ordered pairs $D \subseteq\{(i, j) \mid i, j \in N, i \neq j\}$ is a set of arcs. In this paper, the nodes represent the players in a game, and therefore we refer to the nodes as players. We denote the set of all digraphs by $\mathcal{D}$. For $(N, D) \in \mathcal{D}$, a sequence of $k$ different players $\left(i_{1}, \ldots, i_{k}\right)$ is a (directed) path if $\left(i_{l}, i_{l+1}\right) \in D$ for $l=1, \ldots, k-1$. For $i \in N$, a player $j \in N$ is a subordinate of $i$ if there is a path $\left(i_{1}, \ldots, i_{k}\right)$ with $i_{1}=i$ and $i_{k}=j$. Player $i$ is a superior of $j$ if and only if $j$ is a subordinate of $i$. We denote $F_{D}(i)$ as the set of subordinates of $i$ and $P_{D}(i)$ as the set of superiors of $i$ in $(N, D)$. We also denote $F_{D}^{0}(i)=F_{D}(i) \cup\{i\}$ and $P_{D}^{0}(i)=P_{D}(i) \cup\{i\}$. Also for all $S \subseteq N$ we define $F_{D}(S)=\cup_{i \in S} F_{D}(i)$ and similarly $P_{D}(S)=\cup_{i \in S} P_{D}(i), F_{D}^{0}(S)=\cup_{i \in S} F_{D}^{0}(i)$ and $P_{D}^{0}(S)=\cup_{i \in S} P_{D}^{0}(i)$.

A node $i \in N$ is called a top player in $(N, D)$ if $P_{D}(i)=\emptyset$. A digraph $(N, D)$ is a (directed) rooted tree with root $i$ when (i) player $i$ is the unique top player and (ii) for all $j \neq i$ there is a unique path from $i$ to $j$. In the sequel we denote by $\mathcal{D}_{t}$ the class of rooted trees and an element of $\mathcal{D}_{t}$ by $(N, T)$. Note that for a rooted tree $(N, T)$ with root $i$ it holds that $F_{T}(i)=N \backslash\{i\}$ and for every node $j \neq i$ there is precisely one player $k \in P_{T}(j)$ such that $(k, j) \in T$. This player is called the predecessor of $j$ and denoted by $p(j)$. A player is called a leaf of $(N, T)$ if $F_{T}(i)=\emptyset$. We denote the set of all leafs by $L(T)$. We say that a tree $(N, T)$ is linear if $|L(T)|=1$. In this case it holds that for every player $k$ not in $L(T)$ there is precisely one $h$ such that $(k, h) \in T$. For a tree $(N, T)$ with root $i$, let $M \subseteq N$ be such that (i) $|M| \geq 2$, (ii) $i \in M$, (iii) for every $j \in M \backslash\{i\}$ all nodes on the (unique) directed path from $i$ to $j$ are also in $M$. Then $(M, T(M))$ denotes the subtree of $(N, T)$ restricted to $M$ and $\mathcal{T}$ denotes the collection of all subtrees $(M, T(M))$ of $(N, T)$. 
A permission tree game is a triple $(N, v, T)$ with $N \subset \mathbb{N}$ a finite set of players, $(N, v) \in \mathcal{G}$ a TU-game and $(N, T) \in \mathcal{D}_{t}$ a rooted tree on $N$. In those games, it is assumed that the tree represents a hierarchy that imposes restrictions on the forming of coalitions. Solutions for permission tree games have been discussed in for instance van den Brink, Herings, van der Laan and Talman (2016) and van den Brink, Dietz, van der Laan and Xu (2015). One of these solutions is the permission value, based on the so-called conjunctive approach to permission structures as developed in Gilles, Owen and van den Brink (1992). In this approach, it is assumed that a coalition is feasible if and only if for every player in the coalition all its predecessors are also in the coalition. feasible coalitions is given by

$$
\Phi_{T}=\left\{S \subseteq N \mid P_{T}(i) \subseteq S \text { for all } i \in S\right\}
$$

In this paper we only consider triples $(N, v, T)$ with the permission structure $(N, T) \in \mathcal{D}_{t}$ a rooted tree. We denote by $\mathcal{G}_{\mathcal{T}}$ the collection of all permission tree games. A (single-valued) solution $f$ on $\mathcal{G}_{\mathcal{T}}$ assigns a unique payoff vector $f(N, v, T) \in \mathbb{R}^{N}$ to every $(N, v, T) \in \mathcal{G}_{\mathcal{T}}$. For $S \subseteq N$, let $\sigma_{T}(S)=\bigcup_{R \in \Phi_{T}: R \subseteq S} R$ be the largest feasible subset ${ }^{6}$ of $S$. Following Gilles Owen and van den Brink (1992), the induced permission restricted game of $(N, v, T)$ is the game $\left(N, r_{N, v, T}\right) \in \mathcal{G}$ given by

$$
r_{N, v, T}(S)=v\left(\sigma_{T}(S)\right) \text { for all } S \subseteq N
$$

and the permission value $\psi$ on $\mathcal{G}_{\mathcal{T}}$ is the solution that assigns to every $(N, v, T) \in \mathcal{G}_{T}$ the Shapley value of the associated permission restricted game, thus

$$
\psi(N, v, T)=S h\left(N, r_{N, v, T}\right) \text { for all }(N, v, T) \in \mathcal{G}_{\mathcal{T}}
$$

\section{$3 \quad$ Liability problems with rooted-tree structure}

A liability problem with rooted-tree structure (shortly a liability problem) is a triple $(N, T, d)$ with $(N, T) \in \mathcal{D}_{t}$ a rooted tree on $N$ and $d \in \mathbb{R}_{+}^{N}$ a profile of non-negative damages, with $d_{i}$ the (direct) damage caused by tortfeasor $i \in N$. We denote the class of all liability problems by $\mathcal{L}$. We also define the following notions.

Total damage For $S \subseteq N$, the total damage of $S$ is $d_{S}=\sum_{j \in S} d_{j}$.

Cumulative damage For $S \subseteq N$, the cumulative damage up to $S$ is $c_{S}=\sum_{j \in P_{T}^{0}(S)} d_{j}$. Additional damage For $S \subseteq N$, the additional damage of $S$ is $e_{S}=\sum_{j \in F_{T}^{0}(S)} d_{j}$. Potential damage For every subset $S \subseteq N$, the potential damage of $S$ is $b_{S}=\sum_{j \in S: P_{T}(j) \subseteq S} d_{j}$.

\footnotetext{
${ }^{6}$ Every coalition having a unique largest feasible subset follows from the fact that $\Phi_{T}$ is union closed, i.e. for every $E, F \in \Phi_{T}$ it holds that $E \cup F \in \Phi_{T}$
} 
The total damage of $S$ is the sum of the damages of the players in $S$. The cumulative damage of $S$ is the sum of the damages of the players in $S$ and all their superiors. The additional damage of $S$ is the sum of the damages of the players in $S$ and all their subordinates and can be seen as the sum of all damages that would have been avoided when none of the members of $S$ exercised a wrongful act. The potential damage of $S$ is the sum of all damages that the members of $S$ cause when the members outside $S$ do not behave wrongfully. In case the tree is linear and $S=\{j\}$ for some $j \in N$, these notions coincide with the notions of Dehez and Ferey (2013) as discussed in the Introduction. For ease of notation we denote $d_{S}=d_{i}$ if $S=\{i\}$ and similarly for the other notions. Note that $d_{N}=c_{N}=e_{N}=b_{N}$, and for the root $i, d_{i}=c_{i}=b_{i}$ and $e_{i}=\sum_{j \in F_{T}^{0}(i)} d_{j}=d_{N}$. The following example illustrates the different notions of damages mentioned above.

Example 3.1 Consider six players and rooted-tree $(N, T)$ with $N=\{1,2,3,4,5,6\}$ and $T=\{(1,2),(2,3),(2,4),(1,5),(5,6)\}$, see Figure 2. Table 1 gives the four notions of damages for two different sets $S$. Note that $d_{N}=\sum_{i \in N} d_{i}$.

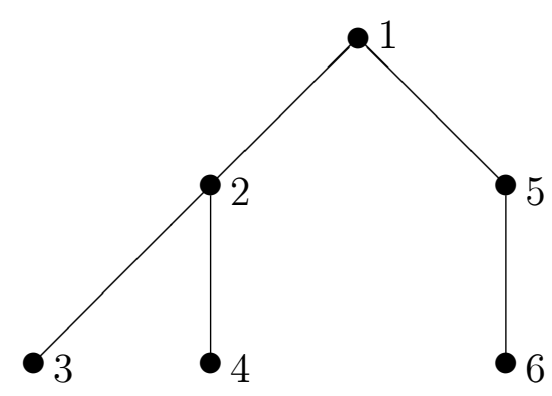

Figure 2: Rooted tree with six players. 
Table 1. Four notions of damages for the tree of Figure 2.

\begin{tabular}{|l||l|l|}
\hline & \multicolumn{1}{|c|}{$S=\{1,4,6\}$} & \multicolumn{1}{|c|}{$S=\{2,6\}$} \\
\hline Total damage $d_{S}$ & $d_{1}+d_{4}+d_{6}$ & $d_{2}+d_{6}$ \\
\hline Cumulative damage $c_{S}$ & $d_{N}-d_{3}$ & $d_{1}+d_{2}+d_{5}+d_{6}$ \\
\hline \hline Additional damage $e_{S}$ & $d_{N}$ & $d_{2}+d_{3}+d_{4}+d_{6}$ \\
\hline Potential damage $b_{S}$ & $d_{1}$ & 0 \\
\hline
\end{tabular}

Given $(N, T, d) \in \mathcal{L}$, an allocation for $(N, T, d)$ is a non-negative vector $x \in \mathbb{R}_{+}^{N}$ such that $\sum_{i \in N} x_{i}=d_{N}$. A compensation scheme for liability problems is a mapping $\varphi$ on $\mathcal{L}$ that associates with every problem $(N, T, d) \in \mathcal{L}$ an allocation $\varphi(N, T, d) \in \mathbb{R}_{+}^{N}$.

We generalize the liability game as defined by Dehez and Ferey (2013) for liability problems with linear structure to the class of liability problems with rooted tree structure. For liability problem $(N, T, d) \in \mathcal{L}$ the corresponding lower-bound liability game is the game $\left(N, v_{L}\right)$ where the worth of a coalition $S$ is its potential damage, i.e. for all $S \subseteq N$,

$$
v_{L}(S)=b_{S}
$$

Dehez and Ferey (2013) focus on the Shapley value of the (lower-bound) liability game. In the sequel we define the Shapley value of a liability problem $(N, T, d)$ as the Shapley value of the corresponding lower-bound liability game. Formally we have the following definition.

Definition 3.2 The Shapley compensation scheme is the mapping Sh on $\mathcal{L}$ that associates with every problem $(N, T, d) \in \mathcal{L}$ the Shapley value of its corresponding lower-bound liability game $\left(N, v_{L}\right)$ :

$$
\operatorname{Sh}(N, T, d) \equiv \operatorname{Sh}\left(N, v_{L}\right) .
$$

In the literature on game theory, the lower-bound liability game is known as the peergroup game associated to peer-group situation $(N, T, d)$, see e.g. Brânzei, Fragnelli and Tijs (2002). A peer-group game, and so the game $\left(N, v_{L}\right)$, is convex. Further it holds that $\left(N, v_{L}\right)$ is the permission restricted game $\left(N, r_{N, v, T}\right)$ of the additive game $(N, v)$ with characteristic function defined as $v(S)=d_{S}$ for all $S \subseteq N$. Therefore, the Shapley value of game $\left(N, v_{L}\right)$ is equal to the permission value of the permission tree game $(N, T, v)$. Since $\left(N, v_{L}\right)$ is convex, its Shapley value is in its core: $\operatorname{Sh}\left(N, v_{L}\right) \in C\left(N, v_{L}\right)$. It follows that $\sum_{i \in S} S h_{i}(N, T, d)=\sum_{i \in S} S h_{i}\left(N, v_{L}\right) \geq v_{L}(S)$ for every $S \subseteq N$ and thus the Shapley compensation scheme satisfies the requirement that for every $S \subseteq N$ the total compensation paid by its members is at least equal to $v_{L}(S)$, being the potential damage of $S$. The 
Shapley compensation scheme thus satisfies Property (i) of the Introduction as required in Dehez and Ferey (2013).

While Dehez and Ferey (2013) focus on the Shapley value of the (lower-bound) liability game, in this paper we consider the nucleolus. We define the nucleolus compensation scheme as the mapping that assigns to every liability problem $(N, T, d)$ the nucleolus of its corresponding lower-bound liability game $\left(N, v_{L}\right)$.

Definition 3.3 The nucleolus compensation scheme is the mapping Nuc on $\mathcal{L}$ that associates with every problem $(N, T, d) \in \mathcal{L}$ the nucleolus of its corresponding lower-bound liability game $\left(N, v_{L}\right)$ :

$$
\operatorname{Nuc}(N, T, d) \equiv N u c\left(N, v_{L}\right)
$$

Since the nucleolus of a game is in its core (if the latter is non-empty), the $\mathrm{Nu}$ cleolus compensation scheme satisfies the requirement that for every $S \subseteq N$ the total compensation paid by its members is at least equal to the potential damage of $S$ $\left(\sum_{i \in S} N u c_{i}(N, T, d)=\sum_{i \in S} N u c_{i}\left(N, v_{L}\right) \geq v_{L}(S)\right.$ for every $\left.S \subseteq N\right)$ and thus respects Property (i) of the Introduction. Nevertheless, we now run into a difficulty about the interpretation of the core and nucleolus. Typically it is considered to be desirable that a payoff vector is in the core of the game. This is called core-stability, saying that every coalition $S$ gets at least its own worth $v(S)$ and so the members of $S$ don't have an incentive to deviate from the grand coalition $N$. However, this holds for profit games in which $v(S)$ is the worth that the members of $S$ can earn by themselves without cooperating with the others, and the entries of $x$ yield payoffs that are paid to the players. In contrast to this usual situation, the Shapley value or the nucleolus of a liability game gives a vector of compensations to be paid by the players in the game, i.e., by the tortfeasors. They are not looking for core stability, on the contrary they want to pay as little as possible.

Therefore, the lower-bound liability game should not be considered as a game that is played by the tortfeasors themselves, but as a model to help the court to determine the compensations to be paid to the injured party. With this interpretation the game gives for every subset (coalition) of tortfeasors, the lower-bounds of their compensations and for instance the Shapley value or the nucleolus can be applied to determine how much every coalition has to pay in addition to its lower bound $v_{L}(S)$. According to the Shapley value, these additional payments are determined by the marginal contributions of the players. ${ }^{7}$ In contrast, the nucleolus is determined by the excesses of the coalitions. However, for a liability game $\left(N, v_{L}\right)$ the excess $e\left(S, x, v_{L}\right)=v_{L}(S)-\sum_{i \in S} x_{i}$ is now a measure of

\footnotetext{
${ }^{7}$ Since the marginal contributions of a player do not depend on the damages of his superiors in the tree, this also implies that the Shapley value satisfies the property that the compensation to be paid by a tortfeasor does not depend on the damages of its superiors, see Ferey and Dehez (2015).
} 
satisfaction of $S$ at $x$, because the bigger the excess is, the lower the total amount of compensation that the members of $S$ have to pay. So, while in a game in which the payoff vector yields payments to the players the nucleolus minimizes lexicographically the vector of dissatisfactions, in the liability game the nucleolus minimizes lexicographically the vector of satisfactions. This is counterintuitive. Even if we consider the game as a model used by the court to determine the compensations, there is no a priori reason to do so.

Nevertheless, the Nucleolus compensation scheme is justified as a reasonable solution when we consider the second requirement of Dehez and Ferey (2013), namely that every tortfeasor should pay at most the additional damage that he would have caused, see Property (ii) in the Introduction. This principle is supported by the third Restatement of Torts as formulated by the American Law Institute. Based on this property, we define for a liability problem $(N, T, d) \in \mathcal{L}$ the corresponding upper-bound liability game as the game $\left(N, v_{U}\right)$ defined by setting, for all $S \subseteq N$,

$$
v_{U}(S)=e_{S},
$$

i.e. the worth of a coalition $S$ is the additional damage that the agents in $S$ might cause. ${ }^{8}$ The next lemma states that $\left(N, v_{U}\right)$ is the dual game of $\left(N, v_{L}\right)$. Because $\left(N, v_{L}\right)$ is convex this implies that $\left(N, v_{U}\right)$ is concave.

Lemma 3.4 For a liability problem $(N, T, d) \in \mathcal{L}$, the upper-bound liability game $\left(N, v_{U}\right)$ is the dual of the lower-bound liability game $\left(N, v_{L}\right)$.

Proof. For $S \subseteq N$, we have that $v_{L}(S)=b_{S}=\sum_{j \in S: P_{T}(j) \subseteq S} d_{j}$. Now, note that

$$
\left\{j \in S: P_{T}(j) \subseteq S\right\}=S \backslash F_{T}(N \backslash S)
$$

i.e., for every $S \subseteq N$, the set of players in $S$ such that all their predecessors are also in $S$ coincides with the set of players in $S$ that are not subordinates of the players in $N \backslash S$. With $F_{T}^{0}(N \backslash S)=F_{T}(N \backslash S) \cup(N \backslash S)$ it follows that $S \backslash F_{T}(N \backslash S)=N \backslash F_{T}^{0}(N \backslash S)$. Hence for every $S \subseteq N$ we obtain that $v_{L}(S)=\sum_{j \in N: j \notin F_{T}^{0}(N \backslash S)} d_{j}$ and thus

$$
\begin{gathered}
v_{L}^{d}(S)=v_{L}(N)-v_{L}(N \backslash S)=\sum_{j \in N} d_{j}-\sum_{j \in N: j \notin F_{T}^{0}(N \backslash(N \backslash S))} d_{j}= \\
\sum_{j \in F_{T}^{0}(N \backslash(N \backslash S))} d_{j}=\sum_{j \in F_{T}^{0}(S)} d_{j}=e_{S}=v_{U}(S) .
\end{gathered}
$$

\footnotetext{
${ }^{8}$ For a liability problem with linear structure the game $\left(N, v_{U}\right)$ is a so-called airport game, see Littlechild and Owen (1973).
} 
We now have the following proposition, where statement (i) follows because the Shapley value is self-dual and the other two statements follow from applying Proposition 2.1 to the convex game $\left(N, v_{L}\right)$ and its dual $\left(N, v_{U}\right)$.

Proposition 3.5 For every liability $\operatorname{problem}(N, T, d) \in \mathcal{L}$ the corresponding games $\left(N, v_{L}\right)$ and $\left(N, v_{U}\right)$ satisfy the following statements:

(i) $\operatorname{Sh}\left(N, v_{L}\right)=\operatorname{Sh}\left(N, v_{U}\right)$,

(ii) $C\left(N, v_{L}\right)=A C\left(N, v_{U}\right)$,

(iii) $\operatorname{Nuc}\left(N, v_{L}\right)=A N u c\left(N, v_{U}\right)$.

Statement (i) implies that the Shapley value of the lower-bound liability game is equal to the Shapley value of the upper-bound liability game. Since $\left(N, v_{L}\right)$ convex and thus $\operatorname{Sh}\left(N, v_{L}\right) \in C\left(N, v_{L}\right)$, statement (ii) implies that $\operatorname{Sh}\left(N, v_{L}\right) \in A C\left(N, v_{U}\right)$, and thus $S h\left(N, v_{L}\right)$ satisfies for every coalition $S$ the upper-bound requirement that $\sum_{i \in S} S h_{i}\left(N, v_{L}\right) \leq$ $e_{S}$. This also holds for $N u c\left(N, v_{L}\right)$. From statement (iii) it follows that $N u c\left(N, v_{L}\right)$ lexicographically maximizes the cost savings $v_{U}(S)-\sum_{i \in S} x_{i}$ with respect to the upper-bound liability game. So, when the court decides to implement $\operatorname{Nuc}\left(N, v_{L}\right)=\operatorname{ANuc}\left(N, v_{U}\right)$, the smallest cost saving over all coalitions $S$ is made as large as possible, then the second smallest is made as large as possible, then the third smallest, and so on. This is a very desirable property and gives a strong motivation for the use of the nucleolus.

We now consider the computation of the compensations for both the Shapley value and the nucleolus. The Shapley value is easy to compute for liability games. Recall that $\left(N, v_{L}\right)$ is the peer-group game associated to the peer-group situation $(N, T, d)$ and also that it is the permission restricted game $\left(N, r_{N, v, T}\right)$ of the additive game $(N, v)$ with its characteristic function defined as $v(S)=d_{S}$ for all $S \subseteq N$. For these games, it is well-known that the Shapley value distributes the damage $d_{i}$ of a player $i \in N$ equally amongst player $i$ and all its superiors in $(N, T)$. This gives the following expression for the compensation to be paid by a tortfeasor $j \in N$ according to the Shapley compensation scheme:

$$
S h_{j}(N, T, d)=\sum_{i \in F_{T}^{0}(j)} \frac{d_{i}}{\left|P_{T}^{0}(i)\right|} .
$$

So, when applying the Shapley value the compensation to be paid by tortfeasor $j$ is the sum of all his shares in the damages of himself and his subordinates.

There is no explicit formula available for the nucleolus, but the nucleolus compensations can be computed by the algorithm given in Brânzei, Solymosi and Tijs (2005) for peergroup games. Given a liability problem $(N, T, d) \in \mathcal{L}$, we assume without loss of generality 
that $1 \in N$ is the root of $(N, T)$. For a subtree $(M, T(M)) \in \mathcal{T}$ (see Section 2), let $(M, T(M), a), a \in \mathbb{R}_{+}^{M}$ be a liability problem on $M$. We define for every $j \in M \backslash\{1\}$,

$$
\tau_{j}(M, T(M), a) \equiv \frac{\sum_{k \in F_{T(M)}^{0}(j)} a_{k}}{\left|F_{T(M)}^{0}(j)\right|+1} .
$$

The Nucleolus compensation scheme is obtained by the following algorithm, where $x=$ $\operatorname{Nuc}(N, T, d) \equiv \operatorname{Nuc}\left(N, v_{L}\right)$. Recall that $p(j)$ is the predecessor of $j$ in $(N, T)$ and so also in every subtree $(M, T(M))$ containing $j$.

\section{Nucleolus algorithm:}

Step 0: Set $M=N$ and $a=d$.

Step 1: Find a $j \in M \backslash\{1\}$ such that $\tau_{j}(M, T(M), a)=\min _{m \in M \backslash\{1\}} \tau_{m}(M, T(M), a)$.

Step 2: For every $k \in F_{T(M)}^{0}(j)$, set $x_{k}=\tau_{j}(M, T(M), a)$. If $\left|M \backslash F_{T(M)}^{0}(j)\right| \geq 2$, go to Step 3. Otherwise, set $x_{1}=d_{N}-\sum_{k \in N \backslash\{1\}} x_{k}$ and stop.

Step 3: Set $M \equiv M \backslash F_{T(M)}^{0}(j)$ and set $a_{p(j)} \equiv a_{p(j)}+x_{j}$. Return to Step 1 .

Note that in Step 2, if $\left|M \backslash F_{T(M)}^{0}(j)\right|=1$, then $M \backslash F_{T(M)}^{0}(j)=\{1\}$. In the next Example we illustrate the above procedure.

Example 3.6 Let $(N, T, d)$ be the liability problem with rooted tree as given in Figure 2 and with vector of damages given by $d=(0,12,40,36,12,30)$.

Using formula (3.1) we have the following computations for the Shapley compensation scheme, starting with the leafs: $S_{3}(N, T, d)=\frac{d_{3}}{3}=\frac{40}{3}, S h_{4}(N, T, d)=\frac{d_{4}}{3}=12$, $S h_{6}(N, T, d)=\frac{d_{6}}{3}=10$. Next we obtain $S h_{2}(N, T, d)=\frac{d_{2}}{2}+S h_{3}(N, T, d)+S h_{4}(N, T, d)=$ $6+12+\frac{40}{3}=\frac{94}{3}, S h_{5}(N, T, d)=\frac{d_{5}}{2}+S h_{6}(N, T, d)=6+10=16$ and finally $S h_{1}(N, T, d)=$ $d_{1}+S_{2}(N, T, d)+S h_{5}(N, T, d)=0+\frac{94}{3}+16=\frac{142}{3}$. Thus $\operatorname{Sh}(N, T, d)=\left(\frac{142}{3}, \frac{94}{3}, \frac{40}{3}, 12,16,10\right)$.

To apply the algorithm for computing the nucleolus payoffs, let $x=\operatorname{Nuc}(N, T, d)$. Then the algorithm performs as follows.

Step 0: Set $M=N$ and $a=d$.

Iteration 1:

Step 1: $\min \left\{\frac{30}{2}, \frac{12+30}{3}, \frac{36}{2}, \frac{40}{2}, \frac{12+40+36}{4}\right\}=14=\tau_{5}(M, T(M), a)$.

Step 2: $x_{5}=x_{6}=14$.

Step 3: Set $M=\{1,2,3,4\}$ and $a=\left(d_{1}+14, d_{2}, d_{3}, d_{4}\right)=(14,12,40,36)$.

Iteration 2:

Step 1: $\min \left\{\frac{36}{2}, \frac{40}{2}, \frac{12+40+36}{4}\right\}=18=\tau_{4}(M, T(M), a)$. 
Step 2: $x_{4}=18$.

Step 3: Set $M=\{1,2,3\}$ and $a=\left(d_{1}+14, d_{2}+18, d_{3}\right)=(14,30,40)$.

Iteration 3:

Step 1: $\min \left\{\frac{40}{2}, \frac{30+40}{3}\right\}=20=\tau_{3}(M, T(M), a)$.

Step 2: $x_{3}=20$.

Step 3: Set $M=\{1,2\}$ and $a=\left(d_{1}+14, d_{2}+18+20\right)=(14,50)$.

Iteration 4:

Step 1: $\tau_{2}(M, T(M), a)=\frac{50}{2}=25$.

Step 2: $x_{2}=25$ and $x_{1}=d_{N}-\sum_{k \in N \backslash\{1\}} x_{k}=39$. Stop.

We have found that $\operatorname{Nuc}(N, T, d)=x=(39,25,20,18,14,14)$.

From formula (3.1) it follows immediately that the Shapley compensation scheme satisfies monotonicity in the sense that for every player $j \in N \backslash L(T)$ it holds that $j$ has to pay at least as much as any of his subordinates (and strictly more when $d_{j}>0$ ). This property also holds for the nucleolus compensation scheme, albeit that compensations can be equal when $d_{j}>0$. We call this weak monotonicity. ${ }^{9}$

\section{Lemma 3.7 Weak Monotonicity}

The compensation scheme $N u$ on the class $\mathcal{L}$ of liability problems satisfies that for every $(N, T, d) \in \mathcal{L}$ it holds for every $j \in N \backslash L(T)$ that

$$
N u c_{j}(N, T, d) \geq N u c_{k}(N, T, d) \text { for every } k \in F_{T}(j) \text {. }
$$

Proof. The proof follows from the algorithm. In the first iteration the algorithm starts with $(N, T, d)$. Let $k \in N \backslash\{1\}$ be the player such that $\tau_{k}(N, T, d)=\min _{j \in N \backslash\{1\}} \tau_{j}(N, T, d)$. Then $N u c_{h}(N, T, d)=\tau_{k}(N, T, d)$ for every $h \in F_{T}^{0}(k)$ and so the property holds for $k$ and all his subordinates. In the second iteration we have $M=N \backslash F_{T}^{0}(k)$ and the reduced liability problem $(M, T(M), a)$ with $a_{p(k)}=d_{p(k)}+\tau_{k}(N, T, d)$, and $a_{h}=d_{h}$ for every $h \in M \backslash\{p(k)\}$. We show that the minimal $\tau_{j}(M, T(M), a)$ in the second iteration is at least equal to $\tau_{k}(N, T, d)$. For simplicity of notation, denote $A_{j}=\tau_{j}(N, T, d)$ and $n_{j}=\left|F_{T}^{0}(j)\right|$ for every $j \in N \backslash\{1\}$.

First, consider a player $h \in M$ that is neither a subordinate of $k$ in $(N, T, d)$, nor a superior of $k$ in $(N, T, d)$. Then $\tau_{h}(M, T(M), a)=\tau_{h}(N, T, d)=A_{h} \geq A_{k}$ (since $A_{k}$ was minimal in the previous iteration). Second, consider a superior $h$ of $k$ in $(N, T, d)$. Now note that in the first iteration $n_{k}$ players ( $k$ and its subordinates) have left and that all these players paid compensation $A_{k}$, while $a_{p(k)}=d_{p(k)}+A_{k}$. Further note that $h$ is

\footnotetext{
${ }^{9}$ This property is called structural monotonicity in van den Brink and Gilles (1996) who use it to axiomatize the conjunctive (Shapley) permission value for permission restricted games.
} 
either $p(k)$ itself, or it is a superior of $p(k)$ and that $A_{h}=\frac{1}{n_{h}+1} \sum_{i \in F_{T}^{0}(h)} a_{i}$. From this and $A_{h} \geq A_{k}$ it follows that

$$
\tau_{h}(M, T(M), a)=\frac{\left(n_{h}+1\right) A_{h}-n_{k} A_{k}}{n_{h}-n_{k}+1} \geq \frac{\left(n_{h}+1\right) A_{h}-n_{k} A_{h}}{n_{h}-n_{k}+1}=A_{h} \geq A_{k} .
$$

So, at the second iteration we have for every $h \in M \backslash\{1\}$ that $\tau_{h}(M, T(M), a)$ is at least equal to the compensation $A_{k}$ assigned to $k$ and each of its subordinates in the first iteration. So, the players that leave in the second iteration have to pay at least the same amount as the players that have left in the first iteration.

Continuing in this way we obtain that the assigned payoffs are non-increasing in the iterations. The result now follows from the fact that for any two players $k$ and $h$ with $k$ a subordinate of $h$, player $h$ gets assigned its payoff either in the same iteration as $k$ or in a later iteration.

\section{Axioms}

In this section, we propose three axioms of a compensation scheme for liability problems with rooted-tree structure. These properties are inspired from the observations concerning tort law and case-system consistency, see Ito (1978). We then prove that the Nucleolus compensation scheme is the unique compensation scheme on the class $\mathcal{L}$ of liability problems with rooted-tree structure that satisfies the three properties. Without loss of generality, we assume that for every $(N, T, d) \in \mathcal{L}$ it holds that $1 \in N$ is the top of root $(N, T)$.

The first axiom takes into account the tort law principle that all tortfeasors are held jointly responsible for the total damage $d_{N}=\sum_{j \in N} d_{j}$, and therefore every tortfeasor should pay a fair share of this total damage. According to this principle the first property sets a uniform lower bound on the compensation to be paid by an individual tortfeasor. The axiom is obtained by considering for tortfeasor $i \in N \backslash\{1\}$ the additional damage $e_{i}=\sum_{k \in F_{T}^{0}(i)} d_{k}$ that has been caused by agent $i$ and his subordinates. Without the wrongful act of agent $i$ 's predecessor $p(i)$, the sequentially wrongful acts by agent $i$ and his subordinates would not have occurred. From this viewpoint, all agents in $F_{T}^{0}(i)$ and $p(i)$ are jointly responsible for the additional damage $e_{i}$ of agent $i$. We assume the case of torts where the causal weights between the agents in $F_{T}^{0}(i)$ and $p(i)$ cannot be determined and therefore a per capita criterion is justified. ${ }^{10}$ For $i \in N \backslash\{1\}$, this yields the per capita

\footnotetext{
${ }^{10}$ For instance, Ni and Wang (2007) use a per capita criterion to introduce the upstream equal responsibility sharing method for polluted river problems.
} 
contribution $\tau_{i}(N, T, d)$ given by

$$
\tau_{i}(N, T, d)=\frac{e_{i}}{\left|F_{T}^{0}(i)\right|+1},
$$

i.e., $\tau_{i}(N, T, d)$ is the equal division of the additional damage $e_{i}$ between $i$, all his subordinates and his predecessor.

Now, the first axiom requires that every tortfeasor $i$ should pay at least the smallest per capita contribution $\min _{j \in N \backslash\{1\}} \tau_{j}(N, T, d)$, i.e., the smallest per capita contribution is considered as a guarantee of every injuring party's compensation for the total damage.

Axiom 1 (Uniform lower bound) A compensation scheme $\varphi$ on $\mathcal{L}$ satisfies the uniform lower bound if for every $(N, T, d) \in \mathcal{L}$ and every $i \in N$,

$$
\varphi_{i}(N, T, d) \geq \min _{j \in N \backslash\{1\}} \tau_{j}(N, T, d)
$$

We stress that this is a very weak lower bound. Note that it does not require that $i$ contributes at least its own per capita contribution, but the minimal per capita contribution over all tortfeasors (except the first tortfeasor). In Example 3.6 it requires that every tortfeasor pays at least the per capita contribution of agent 5, being 14 .

The second axiom puts for every tortfeasor an individual upper bound on his compensation. Also this axiom is obtained by considering for tortfeasor $i \in N \backslash\{1\}$ the additional damage $e_{i}$ that has been caused by agent $i$ and his subordinates. Again this damage would not have occurred without the wrongful act of agent $i$ 's predecessor $p(i)$. From this viewpoint, agent $i$ and his predecessor can be held jointly responsible for the damage $e_{i}$. Assuming again the case of torts, the causal weights between $i$ and $p(i)$ cannot be determined. This yields an equal division of $e_{i}$ between $i$ and $p(i)$. The second axiom requires that every tortfeasor $i \neq 1$ should be held responsible for at most the half of his additional damage $e_{i}$. Since agent 1 has no predecessor, he should be held responsible for at most his full additional damage $e_{1}$, which is equal to the total damage $d_{N}$.

Axiom 2 (Individual upper bounds) A compensation scheme $\varphi$ on $\mathcal{L}$ satisfies the individual upper bounds if for every $(N, T, d) \in \mathcal{L}$ and every $i \in N \backslash\{1\}$,

$$
\varphi_{i}(N, T, d) \leq \frac{1}{2} e_{i}
$$

In Example 3.6 the vector of additional damages is given by $e=\left(e_{1}, e_{2}, e_{3}, e_{4}, e_{5}\right)=$ $(130,88,40,36,42,30)$, so the upper bounds for agents 2 to 6 are, respectively, 44, 20, 18, 21 and 15 . 
The third and last axiom is a type of case system consistency. Given a compensation scheme $\varphi$, consider a liability problem $(N, T, d) \in \mathcal{L}$ and a tortfeasor $i \in L(T)$ (thus a tortfeasor that does not have subordinates). Since such a tortfeasor can be held responsible for at most his own damage $d_{i}$, we may assume that he has to pay at most $d_{i}$. Now consider the related liability problem $\left(N \backslash\{i\}, T(N \backslash\{i\}), d^{i}\right)$, where $d^{i} \in \mathbb{R}^{N}$ is the vector of damages given by $d_{p(i)}^{i}=d_{p(i)}+d_{i}-\varphi_{i}(N, T, d)$ and $d_{k}^{i}=d_{k}$ for every $k \neq p(i)$ in $N \backslash\{i\}$. So, this is the liability problem obtained from $(N, T, d)$ by removing leaf $i$ and by adding the remaining damage $d_{i}-\varphi_{i}(N, d, T)$ not paid by $i$ to the damage $d_{p(i)}$ caused by his predecessor $p(i)$. The consistency axiom requires that the outcome chosen by a compensation scheme for every agent $j \in N \backslash\{i\}$ should be invariant under the departure of a leaf $i \in L(T)$ of the tree.

Axiom 3 (Leaf consistency) A compensation scheme $\varphi$ on $\mathcal{L}$ satisfies leaf consistency if for every $(N, T, d) \in \mathcal{L}$, every $i \in L(T)$ and every $j \in N \backslash\{i\}$

$$
\varphi_{j}\left(N \backslash\{i\}, T(N \backslash\{i\}), d^{i}\right)=\varphi_{j}(N, T, d) .
$$

In Example 3.6, leaf consistency, for example, implies that when agent 6 leaves and pays its nucleolus contribution 14, and we consider the new damage vector where the damage of agent 5 is $d_{5}+d_{6}-14=42-14=28$ and the other damages do not change, and in the structure we just leave agent 6 , then the nucleolus payoffs of the other agents do not change.

\section{Characterization of the Nucleolus compensation scheme}

In this section, we show that there is a unique compensation scheme on the class $\mathcal{L}$ of liability problems that satisfies the Axioms 1-3 and that this is the Nucleolus compensation scheme as defined in Definition 3.2. First, note that for an agent $i \in L(T), e_{i}=d_{i}$, and thus for $i \in L(T)$, Axiom 2 requires that $\varphi_{i}(N, T, d) \leq \frac{1}{2} d_{i}$. The next lemma states that when a compensation scheme satisfies Axiom 3 and the individual upper bound requirement for all leafs, then it also satisfies the individual upper bound requirement for all the other agents. In fact, the condition for the leafs and Axiom 3 imply Axiom 2 (for all agents).

Lemma 5.1 Let $\varphi$ on $\mathcal{L}$ be a compensation scheme that satisfies Axiom 3 and $\varphi_{i}(N, T, d) \leq$ $\frac{1}{2} d_{i}$ for every $i \in L(T)$. Then $\varphi$ satisfies Axiom 2 .

Proof. We prove that the upper bound holds for every other agent not being a leaf. Let $j$ be a player such that $j \in N \backslash(\{1\} \cup L(T))$. Let $d^{\prime} \in \mathbb{R}^{N}$ such that $d_{i}^{\prime}=d_{i}$ for $i \in N \backslash F_{j}^{0}(T)$ 
and $d_{j}^{\prime}=e_{j}-\sum_{k \in F_{T}(j)} \varphi_{k}(N, T, d) \leq e_{j}$. By subsequently applying Axiom 3 for all players in $F_{T}(j)$, it follows that $\left(N \backslash F_{T}(j), T\left(N \backslash F_{T}(j)\right), d^{\prime}\right) \in \mathcal{L}$ and

$$
\varphi_{j}(N, T, d)=\varphi_{j}\left(N \backslash F_{T}(j), T\left(N \backslash F_{T}(j)\right), d^{\prime}\right) .
$$

Since $j$ is a leaf on the subtree $\left(N \backslash F_{T}(j), T\left(N \backslash F_{T}(j)\right)\right.$, it follows that

$$
\varphi_{j}\left(N \backslash F_{T}(j), T\left(N \backslash F_{T}(j)\right), d^{\prime}\right) \leq \frac{1}{2} d_{j}^{\prime} \leq \frac{1}{2} e_{j}
$$

The next theorem states that the Nucleolus compensation scheme is the unique compensation scheme that satisfies Axiom 1 and 3 and the individual upper bounds (of Axiom 2) for the leafs. It is the main step to reach our main theorem.

Theorem 5.2 Let $\varphi$ on the class $\mathcal{L}$ of liability problems be a compensation scheme that satisfies Axioms 1 and 3 and for every $(N, T, d) \in \mathcal{L}$ the individual upper bound requirement $\varphi_{i}(N, T, d) \leq \frac{1}{2} e_{i}$ for every $i \in L(T)$. Then $\varphi(N, T, d)=N u c(N, T, d)$.

Proof. We prove that for every liability problem, the Axioms 1 and 3 and the upper bound requirements for the leafs determine the outcome computed by the Nucleolus algorithm. To simplify notation, let $x=\varphi(N, T, d)$. Without loss of generality, it is assumed that 1 is the root of the tree $(N, T)$. We subsequently consider liability problems with respectively $|N|=2,|N|=3$ and $|N|>3$.

Case 1, $|N|=2$. Consider a liability problem $(N, T, d) \in \mathcal{L}$ with $|N|=2$. Then, by Axiom 1 it follows that $x_{2} \geq \tau_{2}(N, T, d)=\frac{1}{2} d_{2}$ and by the upper bound requirement that $x_{2} \leq \frac{1}{2} e_{2}=\frac{1}{2} d_{2}$. Hence $x_{2}=\frac{1}{2} d_{2}$, and thus $x_{1}=d_{1}+\frac{1}{2} d_{2}$. This is equal to the outcome computed by the Nucleolus algorithm.

Case 2, $|N|=3$. For a liability problem $(N, T, d) \in \mathcal{L}$ with $|N|=3$ there are two possibilities: either $(N, T)$ is a line tree (one branch) or $(N, T)$ is a tree with two branches.

Case 2-1. First, we consider a line tree. Without loss of generality, we assume that $p(2)=1$ and $p(3)=2$. Now either $\frac{d_{3}}{2} \leq \frac{d_{2}+d_{3}}{3}$ or not.

Case 2-1-1, $\frac{d_{3}}{2} \leq \frac{d_{2}+d_{3}}{3}$. By Axiom 1 and the individual upper bound requirement for leaf 3 , it follows that $x_{3}=\frac{d_{3}}{2}$. Next, it follows by Axioms 1 and 3 and the uniform lower bound for leaf 2 in the subtree after removing agent 3 that $x_{2}=\frac{d_{2}+d_{3}-x_{3}}{2}$, and finally $x_{1}=d_{N}-x_{2}-x_{3}$. So $x$ is equal to the outcome computed by the Nucleolus algorithm.

Case 2-1-2, $\frac{d_{3}}{2}>\frac{d_{2}+d_{3}}{3}$. By Axiom 1, we must have that $x_{j} \geq \frac{d_{2}+d_{3}}{3}$ for $j=1,2,3$. The individual upper bound for leaf 3 requires that $x_{3}=\frac{d_{3}}{2}-c$ for some $c \geq 0$, and thus $\frac{d_{3}}{2}-c \geq \frac{d_{2}+d_{3}}{3}$. This yields $c \leq \frac{d_{3}}{6}-\frac{d_{2}}{3}$. By Axioms 1 and 3 and the uniform lower bound for leaf 2 in the subtree after removing agent 3 , it follows that $x_{2}=\frac{d_{2}+d_{3}-x_{3}}{2}$, and thus 
$x_{2}=\frac{d_{2}+d_{3}-x_{3}}{2} \geq \frac{d_{2}+d_{3}}{3}$. Substituting $x_{3}=\frac{d_{3}}{2}-c$ in this inequality gives $c \geq \frac{d_{3}}{6}-\frac{d_{2}}{3}$ and thus $c=\frac{d_{3}}{6}-\frac{d_{2}}{3}$. This implies that $x_{2}=x_{3}=\frac{d_{2}+d_{3}}{3}$ and thus $x_{1}=d_{1}+\frac{d_{2}+d_{3}}{3}$. Again $x$ is equal to the outcome computed by the Nucleolus algorithm.

Case 2-2. When $(N, T)$ is a tree with two branches we have $p(2)=p(3)=1$. Without loss of generality, let $d_{2} \geq d_{3}$. By Axiom 1 and the uniform lower bound for leaf 3 it follows that $x_{3}=\frac{d_{3}}{2}$. By Axiom 3 and case $|N|=2, x_{2}=\frac{d_{2}}{2}$ and thus $x_{1}=d_{1}+\frac{d_{2}}{2}+\frac{d_{3}}{2}$. Also in this case we have that $x$ is equal to the outcome computed by the Nucleolus algorithm.

Case 3, $|N|>3$. We now proceed with induction. Consider a liability problem $(N, T, d)$ with $|N|=n>3$. For any liability problem $\left(N^{\prime}, T^{\prime}, d^{\prime}\right) \in \mathcal{L}$ with $\left|N^{\prime}\right|<n$ we assume that $\varphi\left(N^{\prime}, T^{\prime}, d^{\prime}\right)=\operatorname{Nuc}\left(N^{\prime}, T^{\prime}, d^{\prime}\right)$. To show that then $x=N u c(N, T, d)$ we consider two possibilities, namely player $j$ with minimal $\tau_{j}(N, T, d)$ is either a leaf or not.

Case 3-1. There exists $i \in L(T)$ such that $\tau_{i}(N, T, d)=\frac{d_{i}}{2}=\min _{j \in N \backslash\{1\}} \tau_{j}(N, T, d)$. Take this leaf $i$ (if there are multiple, take an arbitrary one). By Axiom 1 and the individual upper bound for leaf $i$ it follows that $x_{i}=\frac{d_{i}}{2}$. This is also the outcome for $i$ as computed by the Nucleolus algorithm, and thus $x_{i}=N u c_{i}(N, T, d)$. Set $N^{\prime}=N \backslash\{i\}$ and take $d^{\prime} \in \mathbb{R}^{N^{\prime}}$ with $d_{j}^{\prime}=d_{j}$ for $j \in N^{\prime} \backslash\{p(i)\}$ and $d_{p(i)}^{\prime}=d_{p(i)}+d_{i}-x_{i}=d_{p(i)}+d_{i}-N u c_{i}(N, T, d)$.

Consider the lower bound liability game $\left(N, v_{L}\right)$ corresponding to $(N, L, d)$. Then the lower bound liability game corresponding to $\left(N^{\prime}, T\left(N^{\prime}\right), d^{\prime}\right)$ is the game $\left(N^{\prime}, v_{L}^{\prime}\right)$ where $v_{L}^{\prime}$ is given by setting for $S \subseteq N^{\prime}$,

$$
v_{L}^{\prime}(S)= \begin{cases}v_{L}(S \cup\{i\})-N u c_{i}(N, T, d) & \text { if } p(i) \in S, \\ v_{L}(S) & \text { otherwise. }\end{cases}
$$

It can be shown that $\left(N^{\prime}, v_{L}^{\prime}\right)$ is the Davis-Maschler reduced game of $\left(N, v_{L}\right)$ on $N^{\prime}$ with respect to $\operatorname{Nuc}(N, L, d) .{ }^{11} \quad$ By this observation and the fact that the nucleolus satisfies Davis-Maschler consistency ${ }^{12}$, it holds that for every $j \in N^{\prime}$ that $N u c_{j}(N, T, d)=$ $N u c_{j}\left(N^{\prime}, T\left(N^{\prime}\right), d^{\prime}\right)$. Further, for every $j \in N^{\prime}$, we have by the induction hypothesis

\footnotetext{
${ }^{11}$ For a game $(N, v) \in \mathcal{G}$, a vector $x \in \mathbb{R}^{N}$ and non-empty subset $N^{\prime}$ of $N$, the Davis-Maschler reduced game (Davis and Maschler, 1965) on $N^{\prime}$ with respect to $(N, v)$ and $x$ is the game $\left(N^{\prime}, w^{x}\right) \in \mathcal{G}$ defined by setting for all $S \subseteq N^{\prime}$,

$$
w^{x}(S)= \begin{cases}v(N)-\sum_{i \in N \backslash N^{\prime}} x_{i} & \text { if } S=N^{\prime}, \\ \max _{T \subseteq N \backslash N^{\prime}}\left[v(S \cup T)-\sum_{i \in T} x_{i}\right] & \text { if } S \neq N^{\prime}, \emptyset, \\ 0 & \text { if } S=\emptyset .\end{cases}
$$

${ }^{12}$ A solution $f$ on a subclass $\mathcal{G}^{\prime}$ of $\mathcal{G}$ satisfies Davis-Maschler consistency (Davis and Maschler, 1965) if for all $(N, v) \in \mathcal{G}^{\prime}$ and every non-empty $N^{\prime} \subset N$ it holds for $x=f(N, v)$ that the DM reduced game $\left(N^{\prime}, w^{x}\right) \in \mathcal{G}^{\prime}$ and $x_{N^{\prime}}=f\left(N^{\prime}, w^{x}\right)$, where $x_{N^{\prime}} \equiv\left(x_{i}\right)_{i \in N^{\prime}}$.
} 
that $\varphi_{j}\left(N^{\prime}, T\left(N^{\prime}\right), d^{\prime}\right)=N u c_{j}\left(N^{\prime}, T\left(N^{\prime}\right), d^{\prime}\right)$. Hence, for every $j \in N^{\prime}$ it holds that $N u c_{j}(N, T, d)=\varphi_{j}\left(N^{\prime}, T\left(N^{\prime}\right), d^{\prime}\right)$. With Axiom 3, it now follows that for every $j \in N^{\prime}$, $x_{j}=\varphi_{j}\left(N^{\prime}, T\left(N^{\prime}\right), d^{\prime}\right)=N u c_{j}(N, T, d)$.

Case 3-2: For all $i \in L(T)$, it holds that $\min _{j \in N \backslash\{1\}} \tau_{j}(N, T, d)<\tau_{i}(N, T, d)=\frac{d_{i}}{2}$. Let $k$ be such that $\tau_{k}(N, T, d)=\min _{j \in N \backslash\{1\}} \tau_{j}(N, T, d)$ and take some $i \in L(T)$ such that $i \in F_{T}^{0}(k)$, thus $i$ is subordinate of $k$. Since $i \neq k$, we have that also $p(i) \in F_{T}^{0}(k)$. From the Nucleolus algorithm we obtain that

$$
N u c_{i}(N, T, d)=N u c_{p(i)}(N, T, d)=\tau_{k}(N, T, d)
$$

By Axiom 1, we have that $x_{i} \geq \tau_{k}(N, T, d)=N u c_{i}(N, T, d)$. Suppose that $x_{i}>N u c_{i}(N, T, d)$. Set $N^{\prime}=N \backslash\{i\}$ and take $d^{\prime} \in \mathbb{R}^{N^{\prime}}$ with $d_{j}^{\prime}=d_{j}$ for $j \in N^{\prime} \backslash\{p(i)\}$ and $d_{p(i)}^{\prime}=d_{p(i)}+d_{i}-x_{i}$. By Axiom 3, we have that

$$
x_{p(i)}=\varphi_{p(i)}\left(N^{\prime}, T\left(N^{\prime}\right), d^{\prime}\right)
$$

and by the induction hypothesis,

$$
\varphi_{p(i)}\left(N^{\prime}, T\left(N^{\prime}\right), d^{\prime}\right)=N u c_{p(i)}\left(N^{\prime}, T\left(N^{\prime}\right), d^{\prime}\right) .
$$

Take $d^{\prime \prime} \in \mathbb{R}^{N^{\prime}}$ with $d_{j}^{\prime \prime}=d_{j}$ for $j \in N^{\prime} \backslash\{p(i)\}$ and $d_{p(i)}^{\prime \prime}=d_{p(i)}+d_{i}-N u c_{i}(N, T, d)$. Since by assumption $x_{i}>N u c_{i}(N, T, d)$, and thus $d_{p(i)}^{\prime}<d_{p(i)}^{\prime \prime}$, it follows from applying the Nucleolus algorithm that

$$
N u c_{p(i)}\left(N^{\prime}, T\left(N^{\prime}\right), d^{\prime}\right)<N u c_{p(i)}\left(N^{\prime}, T\left(N^{\prime}\right), d^{\prime \prime}\right)
$$

By the fact that the lower bound liability game corresponding to $\left(N^{\prime}, T\left(N^{\prime}\right), d^{\prime \prime}\right)$ is the Davis-Maschler reduced game of $\left(N, v_{L}\right)$ on $N^{\prime}$ with respect to $N u c(N, T, d)$, it follows with Davis-Maschler consistency that

$$
N u c_{p(i)}\left(N^{\prime}, T\left(N^{\prime}\right), d^{\prime \prime}\right)=N u c_{p(i)}(N, T, d)
$$

From the (in)equalities (5.2)-(5.6) it follows that

$$
x_{p(i)}<N u c_{p(i)}(N, T, d)=\tau_{k}(N, T, d)
$$

which contradicts Axiom 1. Therefore, for every $i \in L(T) \cap F_{T}^{0}(k), x_{i}=N u c_{i}(N, T, d)$. For every $i \in L(T) \cap F_{T}^{0}(k)$, let $N^{\prime}=N \backslash\{i\}$. Then it follows similar as above and from Axiom 3 that for every $j \in N^{\prime}, x_{j}=N u c_{j}(N, T, d)$.

Theorem 5.2 shows that if a compensation scheme satisfies Axioms 1 and 3 and the individual upper bound for the leafs, then it must be the nucleolus. On the other hand, we 
argue that the Nucleolus compensation scheme satisfies the Axioms 1, 2 and 3. First, from Lemma 3.7 and the algorithm it follows that the nucleolus compensation scheme satisfies Axiom 1. From the proof of Theorem 5.2 it follows that then Nucleolus compensation scheme satisfies Axiom 3 and the individual upper bound for the leafs. It then follows by Lemma 5.1 that Axiom 2 is satisfied. This yields our main result.

\section{Main result}

A compensation scheme $\varphi$ on the class $\mathcal{L}$ of liability problems satisfies Axioms 1, 2 and 3 if and only if $\varphi(N, T, d)=\operatorname{Nuc}(N, T, d)$.

The main result says that there is a unique compensation scheme supported by tort law bounds on the compensations (Axioms 1 and 2) and a type of case-system consistency (Axiom 3). This compensation scheme assigns to every liability problem the Rawlsian outcome given by the nucleolus. Finally, we check logical independence of the three axioms.

- Let $\varphi^{1}$ be the compensation scheme that assigns to every $(N, T, d) \in \mathcal{L}$ compensations given by $\varphi_{i}^{1}(N, T, d)=\min _{j \in N \backslash\{1\}} \tau_{j}(N, T, d)$ for every $i \neq 1$ and $\varphi_{1}^{1}(N, T, d)=$ $d_{N}-\sum_{i \neq 1} \varphi_{i}^{1}(N, T, d)$. Then $\varphi^{1}$ satisfies Axioms 1 and 2 , but not Axiom 3.

- Let $\varphi^{2}$ be the compensation scheme that assigns to every $(N, T, d) \in \mathcal{L}$ compensations given by $\varphi_{i}^{2}(N, T, d)=d_{i}$, for every $i \in N$. Then $\varphi^{2}$ satisfies Axioms 1 and 3 , but not Axiom 2.

- Let $\varphi^{3}$ be the compensation scheme given by $\varphi^{3}(N, T, d)=S h\left(N, v_{L}\right)$ for every $(N, T, d) \in \mathcal{L}$. Then $\varphi^{3}$ satisfies Axiom 2 and Axiom 3 (see Katsev, 2009), but not Axiom 1.

\section{Concluding remarks}

In this paper we considered the Nucleolus compensation scheme as a compensation scheme for liability problems, where causation of the cumulative injury results from multiple sequences of wrongful acts by different parties. It appears that the Nucleolus compensation scheme of a liability problem can be simply computed by using an algorithm for the nucleolus of a corresponding liability game. A very appealing property of the Nucleolus compensation scheme is that with respect to the additional damages of the coalitions, the smallest cost saving over all coalitions is made as large as possible, then the second smallest is made as large as possible, then the third smallest, and so on.

We also characterized the nucleolus compensation scheme by three axioms: a uniform lower bound, an individual upper bound and an axiom on case-system consistency. All these 
three axioms are derived from stylized facts concerning tort law. As noticed in Section 3, the class of lower-bound liability games is equivalent to the class of peer-group games. For this latter class Katsev (2009, chapter 6) provides a characterization of the Shapley value, namely the Shapley value is the unique efficient allocation rule on the class of peergroup games that satisfies leaf consistency, the weak veto property, top monotonicity and independence of non-subordinates. ${ }^{13}$ All these five axioms (including efficiency) are logically independent. The weak veto property states that player $i$ pays at least the same as any other player when $d_{j}=0$ for every $j \neq i$. Top monotonicity states that top player 1 pays at least the same amount as any other player. Finally independendence of non-subordinates states that if the damage of only one player $i$ changes, then the compensations to be paid by the subordinates of $i$ do not change.

We are now ready to compare the Shapley compensation scheme and the nucleolus compensation scheme by their sets of characterizing axioms. It is easy to verify that also the Nucleolus compensation scheme satisfies the weak veto property and top monotonicity, but not independence of non-subordinates. So, the Nucleolus compensation scheme satisfies all above Shapley axioms, except the independence of non-subordinates. On the other hand the Shapley compensation scheme satisfies leaf consistency and the individual upper bound, but not the uniform lower bound. So, the two solutions have in common that both satisfy leaf consistency, individual upper bound, the weak veto property and top monotonicity. Then the Nucleolus compensation scheme satisfies uniform lower bound but not independence of non-subordinates, whereas the Shapley compensation scheme satisfies the latter but not the former. Although the two solutions differ in only one axiom, the differences in the actual outcome for a specific liability problem might be quite big. When $i$ is a leaf of the tree, then equation (3.1) shows that according to the Shapley compensation scheme the damage $d_{i}$ is equally shared by $i$ and all its superiors. In particular, in a linear tree with $n$ tortfeasors the last one pays only a share $\frac{1}{n}$ of its own damage $d_{i}$. On the other hand, according to the Nucleolus compensation scheme the share of a leaf $i$ in its own damage $d_{i}$ can be $\frac{1}{2}$ and this indeed happens when $d_{i}$ is relatively small compared to the damages of its superiors. In fact, for a linear tree with $n$ tortfeasors the uniform lower bound implies that the damage $d_{n}$ of leaf $n$ is shared equally amongst all tortfeasors when $d_{j}=0$ for every superior $j$ of leaf $n$, whereas together with the individual upper bound it also implies that $n$ pays half of its own damage when $d_{n}$ is small compared to the other damages. So, while according to the Shapley compensation leaf $n$ is always held responsible for precisely share $\frac{1}{n}$ of its own damage, according to the Nucleolus compensation scheme

\footnotetext{
${ }^{13}$ Also in Ferey and Dehez (2015) an axiomatization of the Shapley value on the class of (linear) lowerbound liability games is given. In fact, in this axiomatization leaf consistency, the weak veto property and top monotonicity are replaced by the single axiom of zero immediate damage, saying that a player $i$ pays the same as its predecessor $p(i)$ if the damage of the predecessor is equal to zero.
} 
the responsibility of a leaf for its own damage is $\frac{1}{2}$ when his damage is small, but it might decrease to a share of only $\frac{1}{n}$ (as in the Shapley compensation scheme) when his damage is large compared to the damages of his superiors. In fact, we have seen in Section 3 that both the Shapley compensation scheme and the nucleolus compensation scheme satisfy weak monotonicity, saying that a tortfeasor never pays a higher compensation than his predecessor.

Finally, we discuss what happens when the population of the tortfeasors is increasing. Suppose that a new tortfeasor arrives at the end of a branch of the tree, so he is added as new leaf to one of the leafs of the existing tree. One may wonder whether every original tortfeasor pays in the new situation at least the same as in the original situation. If the answer for this question is negative, then it might be that some of the original tortfeasors have an incentive to increase the population of the tortfeasors, which leads to an increase of the total damage. From this aspect, it is appropriate to require that a compensation scheme satisfies leaf population monotonicity, stating that when a new tortfeasor arrives, in the new situation every original tortfeasor pays at least the same as in the original situation. If this property is satisfied, then no original tortfeasor has an incentive to increase the population of the tortfeasors.

From equation (3.1) it follows immediately that the Shapley value satisfies the leaf population property. When a new tortfeasor arrives, then his (additional) damage is equally shared amongst himself and his superiors with no effect on how the other damages are shared. So, the question is whether also the Nucleolus compensation scheme satisfies leaf population monotonicity. This answer is affirmative and follows from a result in Katsev (2009, chapter 6), who shows that when $d$ and $d^{\prime}$ are such that $d_{i}^{\prime} \geq d_{i}$ for every $i \in N$, then every tortfeasor should pay at $d^{\prime}$ at least the same as at $d$. So, for a given tree with a fixed set of tortfeasors, the compensations to be paid by the tortfeasors are non-decreasing in the damages. ${ }^{14}$ Now, suppose a new tortfeasor is added. Then, according to the Nucleolus compensation scheme this tortfeasor pays at most half of its damage and leaf consistency says that the others have to pay according to the original situation, but with the damage of the predecessor of the new leaf replaced by the sum of its own damage and the remaining part of the damage of the new tortfeasor. So, the new situation reduces to the old situation, but with higher damage for the predecessor of the new leaf. So, by the result of Katsev it follows that every original tortfeasor pays at least the same as before. Hence also the Nucleolus compensation scheme satisfies leaf population monotonicity.

\footnotetext{
${ }^{14}$ In the literature this is known as resource monotonicity, but here the resources are the damages.
} 


\section{References}

[1] Boston, G. W. "Apportionment of harm in tort law: a proposed restatement." University of Dayton Law Review 21, 267-378. 1995-1996.

[2] Brânzei, R, V. Fragnelli, S. Tijs. "Three connected line graph peer group situations and line graph peer group games." Mathematical Methods of Operations Research, 55, 93-106, 2002.

[3] Brânzei, R, T. Solymosi, S. Tijs. "Strongly essential coalitions and the nucleolus of peer group games." International Journal of Game Theory, 33, 447-460, 2005.

[4] Brink, R, van den. "An axiomatization of the Shapley value using a fairness property." International Journal of Game Theory 30, 309-319, 2001.

[5] Brink, R, van den, and R.P. Gilles. "Axiomatizations of the conjunctive permission value for games with permission structures." Games and Economic Behavior 12, 113126.

[6] Brink, R, van den, P.J.J. Herings, G. van der Laan, A.J.J. Talman. "The average tree permission value for games with permission structure." Economic Theory 58, 99-123, 2015.

[7] Brink, R, van den, C. Dietz, G. van der Laan, G. Xu. "Comparable characterizations of four solutions for permission tree games." Tinbergen Institute Discussion Paper TI 2015-021/II, 2015. (forthcoming in Economic Theory).

[8] Davis, M, M. Maschler. "The kernel of a Cooperative Game." Naval Research Logistics Quarterly 12, 223-259, 1965.

[9] Dehez, P, S. Ferey. "How to share joint liability: a cooperative game approach." Mathematical Social Sciences 66, 44-50, 2013.

[10] S. Ferey, P. Dehez. "Multiple causation, apportionment and the Shapley value." Core Discussion Paper 2015/16, 2015.

[11] Gilles, R. P, G. Owen, R. van den Brink. "Games with permission structures: the conjunctive approach." International Journal of Game Theory 20, 277-293, 1992.

[12] Ito, M. "An introductory remark." Jurist 59, 6-7, 1978. (in Japanese)

[13] Kalai, E, D. Samet. "On weighted Shapley values." International Journal of Game Theory 16, 205-222, 1987. 
[14] Katsev, I. Solutions for Games with Restricted Cooperation. Ph D. thesis, Free University, Amsterdam, 2009.

[15] Landes, W.M, R.A. Posner. "Joint and multiple tortfeasors: an economic analysis". Journal of Legal Studies 9, 517-555, 1980.

[16] Littlechild S.C, G. Owen. "A simple expression for the Shapley value in a special case". Management Science 3, 370-372, 1973.

[17] Moulin H.V. Fair Division and Collective Welfare. MIT Press, 2003.

[18] Myerson, R.B. "Conference structure and fair allocation rules." International Journal of Game Theory 9, 169-182, 1980.

[19] Ni, D, Y. Wang. "Sharing a polluted river". Games and Economic Behavior 60: 176$186,2007$.

[20] Oishi, T, M. Nakayama. "Anti-dual of economic coalitional TU games". Japanese Economic Review 60, 560-566, 2009.

[21] Oishi, T, M. Nakayama, T. Hokari, Y. Funaki. "Duality and anti-duality in TU games applied to solutions, axioms, and axiomatizations". Journal of Mathematical Economics 63, 44-53, 2016.

[22] Parisi, F, R. Singh. "The efficiency of comparative causation". Review of Law and Economics 6, 219-245, 2010.

[23] Rawls, J. A Theory of Justice, Oxford University Press, New York and Oxford, 1971.

[24] Schmeidler, D. "The nucleolus of a characteristic function game." SIAM Journal on Applied Mathematics 17, 1163-1170, 1969.

[25] Shapley L.S. "A value for $n$-person games." In: Kuhn H, Tucker A.W. (eds) Contributions to the theory of games II, Princeton University Press, pp.307-317, 1953.

[26] Shavell, S. "Torts in which victim and injurer act sequentially". Journal of Law and Economics 26, 589-612, 1983. 